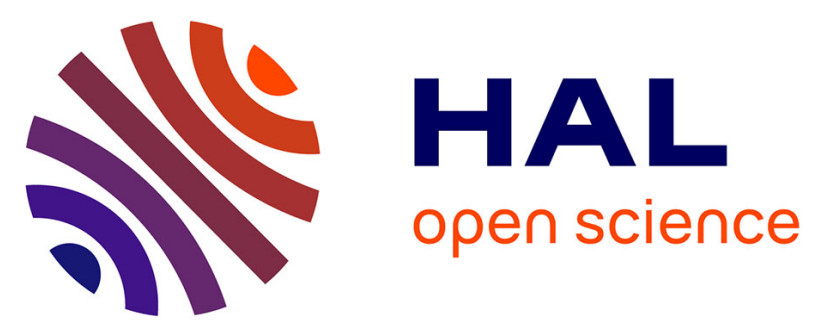

\title{
A multiple level-set approach to prevent numerical artefacts in complex microstructures with nearby inclusions within XFEM
}

A. B. Tran, Julien Yvonnet, Qi-Chang He, C. Toulemonde, J. Sanahuja

\section{To cite this version:}

A. B. Tran, Julien Yvonnet, Qi-Chang He, C. Toulemonde, J. Sanahuja. A multiple level-set approach to prevent numerical artefacts in complex microstructures with nearby inclusions within XFEM. International Journal for Numerical Methods in Engineering, 2011, 85 (11), pp.1436-1459. 10.1002/nme.3025 . hal-00692223

\section{HAL Id: hal-00692223 \\ https://hal.science/hal-00692223}

Submitted on 7 Sep 2014

HAL is a multi-disciplinary open access archive for the deposit and dissemination of scientific research documents, whether they are published or not. The documents may come from teaching and research institutions in France or abroad, or from public or private research centers.
L'archive ouverte pluridisciplinaire HAL, est destinée au dépôt et à la diffusion de documents scientifiques de niveau recherche, publiés ou non, émanant des établissements d'enseignement et de recherche français ou étrangers, des laboratoires publics ou privés. 


\title{
A multiple level-set approach to prevent numerical artefacts in complex microstructures with nearby inclusions within XFEM
}

\author{
A.B. $\operatorname{Tran}^{1}$, J. Yvonnet ${ }^{1, * \dagger}$, Q-C. $\mathrm{He}^{1}$, C. Toulemonde ${ }^{2}$, J. Sanahuja ${ }^{2}$ \\ ${ }^{1}$ Université Paris-Est, Laboratoire Modélisation et Simulation Multi Échelle, MSME UMR 8208 CNRS, 5 bd \\ Descartes, F-77454 Marne-la-Vallée, France. \\ ${ }^{2}$ EDF RED - Département MMC \\ Site des Renardières - Avenue des Renardières - Ecuelles, 77818 Moret sur Loing Cedex, France
}

\section{SUMMARY}

In this paper, we highlight that, when the eXtended Finite Element Method (XFEM) is employed to model a microstructure in which inclusions are involved and the distance between two inclusions is small enough to be comparable with the mesh size, three numerical artefacts are induced, significantly affecting the convergence and accuracy of the numerical solution to the problem with such a microstructure. These artefacts are: a) an artificial percolation of nearby inclusions; b) an artificial distortion of phase domains; c) an enrichment deficiency. We propose to improve the XFEM/Levelset method so as to avoid these artefacts. The new technique leading to this improvement uses one level-set function for each inclusion and adds additional enrichment in an element whose support is cut by several interfaces. A local description of the multiple level-sets is provided to avoid the storage of all level-set functions. A simple integration rule is employed for numerical quadrature in elements cut by several interfaces. We show that the artefacts mentioned hereinbefore are circumvented in this framework. The performances of the method are demonstrated through benchmarks and examples applied to the homogenization of concrete materials in 2D and 3D cases.

Copyright (c) 2000 John Wiley \& Sons, Ltd.

KEY WORDS: Extended Finite Element Method, Level-set, Microstructures, Percolation, Multiple Level-Sets.

\section{INTRODUCTION}

Many materials such as concrete are strongly heterogeneous and contain high volume fractions of inclusions with sizes spread over several spatial scales. Regarding the intensive use of these materials, predicting and optimizing their effective properties from a microscopic description

* Correspondence to: J. Yvonnet, Université Paris-Est Marne-la-Vallée, Laboratoire de Mécanique et Simulation Multi-échelles (MSME), FRE 3160 CNRS, 5 Bd Descartes, 77454 Marne-la-Vallée, France.

${ }^{\dagger}$ Email: julien.yvonnet@univ-paris-est.fr

Contract/grant sponsor: Publishing Arts Research Council; contract/grant number: 98-1846389

Copyright (c) 2000 John Wiley \& Sons, Ltd. 
represents a considerable industrial interest. In view of the microscopic morphological complexity, analytical homogenization approaches are limited, even in the linear case, to bounds of the effective properties. Numerical homogenization techniques allow overcoming these issues, but efficient strategies must be developed to avoid important computational costs, especially in the case of complex three-dimensional models. Many numerical approaches have been proposed over the last decades, mainly based on the finite element method. A straightforward choice is the explicit meshing of all heterogeneities (see e.g. [2, 1, 12]). It requires sophisticated three dimensional mesh softwares, and large computational times related to the meshing operation. Another option is to project the phase properties on a non-conforming mesh, without explicitly representing the interfaces (see e.g. [28, 24]). While avoiding the burden of meshing the heterogeneities surfaces, this method requires fine volume meshes to provide a reasonable description of the local fields in the vicinity of the interfaces. More recently, the use of the Extended Finite Element Method (XFEM) [4, 16, 21] has been proposed for modeling and computing the overall properties of complex microstructures [17]. A major advantage is to describe the interfaces implicitly through a level-set function, with an enriched approximation of the finite element scheme to accurately model the different jumps at the interfaces. In this context, it is also possible to model moving interfaces in fixed meshes [15], cracks which do not coincide with the mesh $[22,6,13]$, or surface and imperfect interfaces effects in composite materials [25, 26, 27]. Recent advances within XFEM have been devoted to prescribe constraints on the interface $[11,18,19,3]$ or to the treatment of issues related to blending elements $[9,10]$.

In complex materials with high volume fraction of inclusions, such as concrete, the explicit meshing of all heterogeneities is a challenging task, due to a large range of characteristic dimensions and closeness of heterogeneities. However, the use of the XFEM/level-set method with a single level-set function to construct the enrichment function induces several difficulties for this class of problems. In this paper, we highlight different numerical artefacts induced by the presence of nearby inclusions within XFEM. It yields a poor description of the local fields as well as a slow convergence of the effective properties of the material with respect to the mesh size. To overcome these limitations, we propose a modified XFEM/Level-set technique which removes the aforementioned issues: each inclusion is associated with a single level-set function and additional degrees of freedom are introduced for nodes whose support is cut by more than one interface. The layout of this paper is as follows.

First, we recall the basic principles of the XFEM/level-set method in section 2 . We then describe the related different artefacts in section 3 in the case of microstructures involving nearby inclusions. Section 4 presents the details of the proposed approach, coined as Multiple Level-set eXtended Finite Element Method ( $\mu$-XFEM) which allows removing the different numerical artefacts, as shown in section 5. Finally, different numerical examples are presented in section 6 to demonstrate the advantage of this approach over the single level-set XFEM approach in such situation. Benchmarks to applications involving three-dimensional models of concrete representative volume element are presented in the small strains elastostatics context.

\section{BASICS OF XFEM/LEVEL-SET}

Modeling a domain containing interfaces with the finite Element Method (FEM) requires a mesh conforming with all internal surfaces. This operation may be highly challenging for 


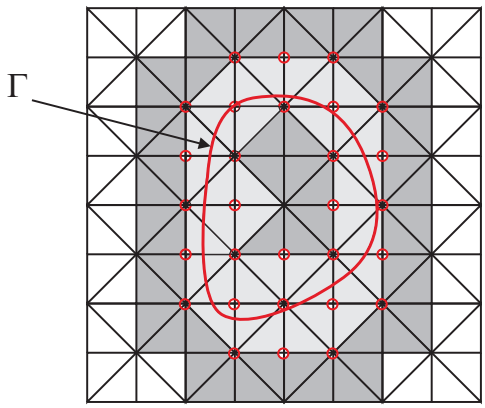

(a)
Enriched elements

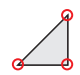

Blending elements

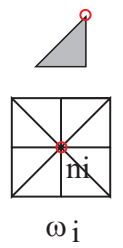

(b)

Figure 1. Interface $\Gamma$ in a non-conforming mesh. Nodes $n_{i}$ whose support $\omega_{i}$ is cut by the interfaces are enriched (denoted by a circle). Elements with all enriched nodes are indicated in clear grey. Partially enriched elements (blending elements) are filled with dark grey.

complex three-dimensional geometries. Examples of such problems can be found in crack propagation, phase evolution or microstructure modeling. In contrast, the XFEM aims to avoid the mesh generation issue by not requiring the finite element mesh to conform to interfaces. The essence of the method is to enrich the finite element approximation with additional functions to model interfaces or singularities independently of the background mesh. The idea follows the Partition of Unity framework [14] by multiplying the enrichment functions that possess desirable approximation by the nodal shape functions.

Consider a domain $\Omega \subset \mathbb{R}^{D}$ ( $D$ being the dimension of the domain) which is partitioned into finite elements, and let $\mathcal{S} \subset \mathbb{N}^{*}\left(\mathbb{N}^{*}\right)$ being the set of positive natural numbers) be a set of $N$ nodes indices in the mesh. Let $\omega_{i}=\operatorname{supp}\left(n_{i}\right)$ being the support of the nodal shape function $N_{i}$, which consists of the union of all elements connected to the node $n_{i}$ (see figure $1 \mathrm{~b}$ ))

The XFEM displacement approximation can be expressed by:

$$
\mathbf{u}^{h}(\mathbf{x})=\sum_{i \in \mathcal{S}} N_{i}(\mathbf{x}) \mathbf{u}_{i}+\sum_{j \in \mathcal{S}^{e}} N_{j}^{*}(\mathbf{x}) \psi(\mathbf{x}) \mathbf{a}_{j}
$$

In the above equation, $\mathbf{u}_{i}$ and $\mathbf{a}_{j}$ are nodal unknowns, $N_{i}(\mathbf{x})$ and $N_{j}^{*}(\mathbf{x})$ are finite element shape functions, not necessarily the same. The nodal set $\mathcal{S}^{e}$ is defined as

$$
\mathcal{S}^{e}=\left\{j \mid j \in \mathcal{S}, \omega_{j} \cap \Gamma \neq \emptyset\right\}
$$

where $\Gamma$ is an interface, that does not necessarily coincide with the mesh. The function $\psi(\mathbf{x})$ is an enrichment function with the desirable discontinuous properties.

If the enrichment function $\psi(\mathbf{x})$ does not vanish on the boundary of $\tilde{\Omega}$ defined as the union of all enriched elements, some of them (called blending elements) are partially enriched (see figure 1) and the function $\psi(\mathbf{x})$ cannot be accurately represented. To avoid this issue, two options are possible. First, we can construct an enrichment function $\psi(\mathbf{x})$ that does vanish on the boundary of $\tilde{\Omega}[17]$. Another possible choice is to use a corrected approximation [9], [10] where a ramp function is constructed in blending elements. In the present paper we will only consider weak discontinuities that can be conveniently reproduced by Eq. (1) with the enrichment function 


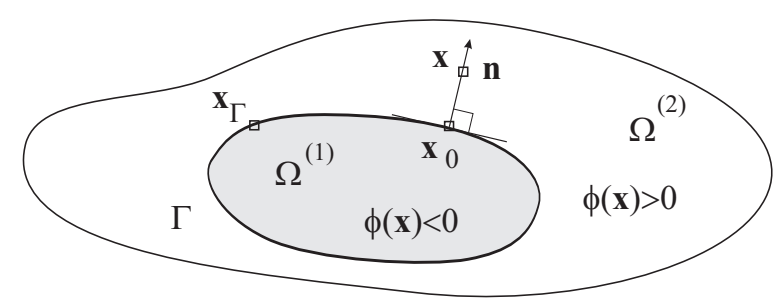

Figure 2. A domain including an interface $\Gamma$. The related level-set function is negative inside $\Gamma$ and positve elsewhere.

proposed in [17]. Further, we will only consider the case $N_{j}^{*}=N_{j}$. An elegant methodology to construct the enrichment function is the level-set method, briefly described as follows.

The level-set method [20] is a numerical technique for tracking moving interfaces. It is based on the idea of representing a level-set curve of a high-dimensional function $\phi(\mathbf{x}, t)$. The concept is closely related to a general method in differential geometry (see e.g. Do Carmo [7], Thorpe $[23])$. In the case of static interfaces, the two ideas coincide. In this paper, we consider only static interfaces.

An interface $\Gamma(\mathbf{x}) \subset \mathbb{R}^{D}$ can be formulated as the level-set curve of a function $\phi: \mathbb{R}^{D} \rightarrow \mathbb{R}$ where

$$
\Gamma(\mathbf{x})=\left\{\mathbf{x} \in \mathbb{R}^{D} \mid \phi(\mathbf{x})=0\right\} .
$$

One important example of such function would be the signed distance function:

$$
\phi(\mathbf{x})=s(\mathbf{x}) \min _{\mathbf{x}_{\Gamma}}\left\|\mathbf{x}-\mathbf{x}_{\Gamma}\right\|=s(\mathbf{x})\left\|\mathbf{x}-\mathbf{x}_{0}\right\|,
$$

where $s(\mathbf{x})= \pm 1$, the sign being chosen arbitrarily negative in $\Omega^{(1)}$ and positive in $\Omega^{(2)}$ (see figure 2). We define the unit vector $\mathbf{n} \equiv \mathbf{n}\left(\mathbf{x}_{0}\right)$ normal to $\Gamma$ at $\mathbf{x}_{0}$ and directed from $\Omega^{(1)}$ to $\Omega^{(2)}$.

Then, $s(\mathbf{x})$ in Eq. (4) can be expressed by:

$$
s(\mathbf{x})=\operatorname{sign}\left(\mathbf{n}\left(\mathbf{x}_{0}\right) \cdot\left(\mathbf{x}-\mathbf{x}_{0}\right)\right)=\mathbf{n}\left(\mathbf{x}_{0}\right) \cdot \frac{\left(\mathbf{x}-\mathbf{x}_{0}\right)}{\left\|\mathbf{x}-\mathbf{x}_{0}\right\|} .
$$

In the case of several interfaces $\Gamma^{k}$ with $k=1,2, \ldots, N_{\text {int }}$ we can define for each associated region $\Omega^{(1) k}$ such as $\Omega^{(1)}=\bigcup_{k}\left\{\Omega^{(1) k}\right\}$ a function

$$
\phi^{k}(\mathbf{x})=s^{k}(\mathbf{x}) \min _{\mathbf{x}_{\Gamma}^{k}}\left\|\mathbf{x}-\mathbf{x}_{\Gamma}^{k}\right\|=s^{k}(\mathbf{x})\left\|\mathbf{x}-\mathbf{x}_{0}^{k}\right\|,
$$

where

$$
s^{k}(\mathbf{x})=\operatorname{sign}\left(\mathbf{n}\left(\mathbf{x}_{0}^{k}\right) \cdot\left(\mathbf{x}-\mathbf{x}_{0}^{k}\right)\right) .
$$

Then the level-set function is finally given by

$$
\phi(\mathbf{x})=\min _{k=1,2, \ldots, N_{\text {int }}} \phi^{k}(\mathbf{x}) .
$$

A simple example that will be considered in this work is the case of several spherical inclusions in 3D or circular inclusions in 2D. In that case, choosing $\Omega^{(1) k}$ as the domain contained within each sphere $k$, Eq. (8) particularizes to 


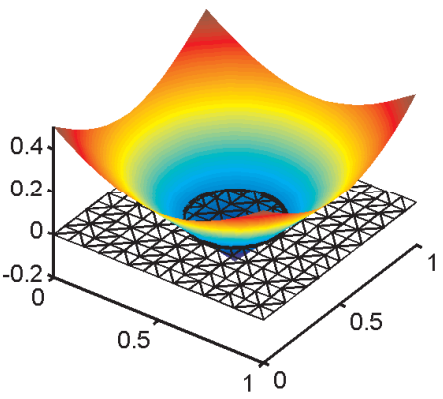

a)

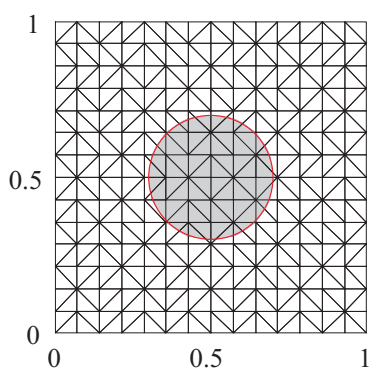

b)

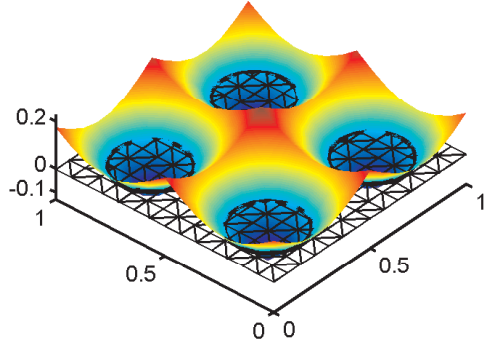

c)

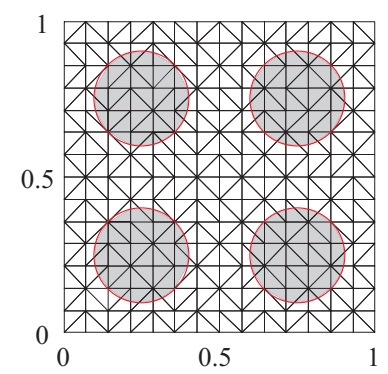

d)

Figure 3. a) Level-set function defining a circular interface; b) mesh and approximated interface associated with the level-set function; c) level-set function defining four circular interfaces; d) mesh and approximated interface associated with the level-set function.

$$
\phi(\mathbf{x})=\min _{k=1,2, \ldots, n_{c}}\left\{\left\|\mathbf{x}-\mathbf{x}_{c}^{k}\right\|-r^{k}\right\}
$$

where $n_{c}$ is number of spherical inclusions, $\mathbf{x}_{c}^{k}$ is the center of inclusion $k$ and $r^{k}$ is the radius of inclusion $k$. An example of level-set function for one and four inclusions is provided in figure 3. An extension of this definition to general shapes, from ellipsoid to parallelepipeds can be found for example in Yvonnet et al. [26].

Sukumar et al. [21] were the first ones to combine the XFEM with a level-set method to construct the enrichment function related to interfaces. Two types of interfaces are generally considered: a) interfaces implying weak discontinuities of the field, i.e. continuous field approximation and discontinuous normal derivatives across $\Gamma$ and b) strong discontinuities related to a field discontinuous approximation across $\Gamma$. In this work we will only consider the first case. Then, as mentioned above, $\psi(\mathbf{x})$ can be chosen in the form proposed by Moës et al. [17] and which does not require a special treatment for blending elements:

$$
\psi(\mathbf{x})=\sum_{i}\left|\phi_{i}\right| N_{i}(\mathbf{x})-\left|\sum_{i} \phi_{i} N_{i}(\mathbf{x})\right| .
$$

To carry out numerical integration, elements cut by the interfaces must be subdivided [21]. If a linear approximation is used for the zero level-set approximation, the interface will result 


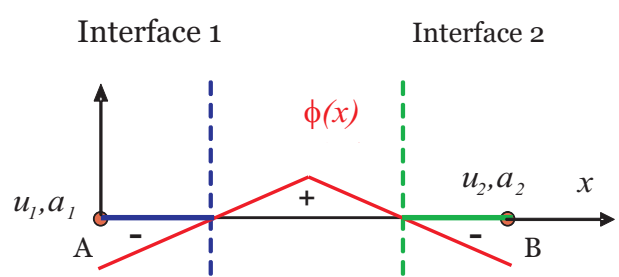

a)

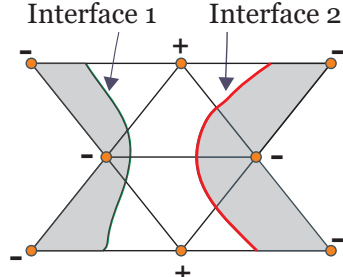

b)

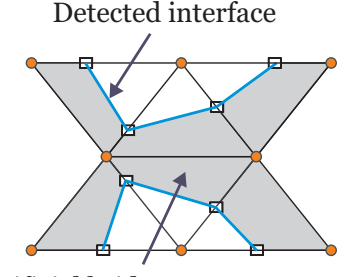

artificial bridge

Figure 4. a) One element containing two interfaces and level-set function related to interfaces; b) theoretical interfaces of two nearby inclusions and background mesh. The "+" and "-" indicate the sign of the level-set nodal values; c) Linear approximation of the zero-level-set.

in piece-wise linear geometrical elements (segments in 2D, triangles in $3 \mathrm{D}$ ). Let $\phi_{i}$ and $\phi_{j}$ denoting nodal level-set values at two vertices $\mathbf{x}_{i}$ and $\mathbf{x}_{j}$ of an element. An element edge is cut by the zero level-set if $\phi_{i} \phi_{j}<0$. The resulting intersection point $\mathbf{x}_{p}$ is then found by

$$
\mathbf{x}_{p}=\mathbf{x}_{i}+\xi\left(\mathbf{x}_{j}-\mathbf{x}_{i}\right), \quad \xi=-\frac{\phi_{i}}{\phi_{j}-\phi_{i}}
$$

In the following, we call "XFEM" the above approximation scheme or any other modified enriched approximation using a single level-set to construct the enrichment function. An alternative is proposed in the following, employing several level-set functions.

\section{NUMERICAL ARTEFACTS}

In this section, we show that several numerical artefacts can be produced by XFEM with a single level-set related to the enrichment function to describe a microstructure in which the distance between two inclusions is comparable with the typical size of the finite elements used.

\subsection{Artificial percolation}

The first artefact concerns the artificial percolation of two inclusions (or inhomogeneities) which are close to each other. Precisely, whenever the surfaces of at least two inclusions in a microstructure simultaneously cut one element, a bridge is created between the two inclusions (see figure $4 \mathrm{~b}$ ) and c)). This is because the nodal values of the level-set function do not change from an inclusion to another, if a single element is cut by at least two interfaces. To explain this point, let us consider a one-dimensional (1D) element containing two interfaces (see figure 4 a)) and let us use a single level-set function $\phi$ which is positive when evaluated at a point within the central domain limited by the interfaces and negative when evaluated at a point outside the central domain. Since the product of the nodal values $\phi_{A}$ and $\phi_{B}$ of $\phi$ at nodes $A$ and $B$ is such that $\phi_{A} \phi_{B}>0$, the presence of the interfaces inside the element cannot be detected.

We observe that the artificial percolation of inclusions in a microstructure occurs when the mesh size is of the order of the distance between inclusions. As will be illustrated in the numerical examples, this artefact can significantly affect the degree of accuracy with which the 
effective properties of heterogeneous materials with inclusions are computed, especially when the property contrast of constituent phases is high.

\subsection{Artificial interface distortion}

When the support of a node is cut by at least two interfaces (see figure 5), the linear interpolation of a single level-set function causes an artificial distortion of the domains delimited by the interfaces. To illustrate this point, we consider a $1 \mathrm{D}$ example described in figure 5 a). A region is discretized by 6 elements defined by the nodal coordinates $X_{1}, X_{2}, \ldots$, $X_{7}$. We are interested in two inclusions of radius $r$, whose centers $X_{C_{1}}$ and $X_{C_{2}}$ are such that $X_{2}<X_{C_{1}}<X_{3}$ and $X_{5}<X_{C_{2}}<X_{6}$.

Using (9), the nodal values of the level-set function $\phi$ are given by

$$
\left\{\begin{array}{l}
\phi\left(x_{i}\right)=\phi^{1}=\min \left(\left|x_{1}-x_{C_{1}}\right|-r,\left|x_{1}-x_{C_{2}}\right|-r\right)=x_{C_{1}}-x_{1}-r, \\
\phi\left(x_{2}\right)=\phi^{2}=\min \left(\left|x_{2}-x_{C_{1}}\right|-r,\left|x_{2}-x_{C_{2}}\right|-r\right)=x_{C 1}-x_{2}-r, \\
\phi\left(x_{3}\right)=\phi^{3}=\min \left(\left|x_{3}-x_{C_{1}}\right|-r,\left|x_{3}-x_{C_{2}}\right|-r\right)=x_{3}-x_{C_{1}}-r, \\
\phi\left(x_{4}\right)=\phi^{4}=\min \left(\left|x_{4}-x_{C_{1}}\right|-r,\left|x_{4}-x_{C_{2}}\right|-r\right)=x_{C_{2}}-x_{4}-r, \\
\phi\left(x_{5}\right)=\phi^{5}=\min \left(\left|x_{5}-x_{C_{1}}\right|-r,\left|x_{5}-x_{C_{2}}\right|-r\right)=x_{C_{2}}-x_{5}-r, \\
\phi\left(x_{6}\right)=\phi^{6}=\min \left(\left|x_{6}-x_{C_{1}}\right|-r,\left|x_{6}-x_{C_{2}}\right|-r\right)=x_{6}-x_{C_{2}}-r, \\
\phi\left(x_{7}\right)=\phi^{7}=\min \left(\left|x_{7}-x_{C_{1}}\right|-r,\left|x_{7}-x_{C_{2}}\right|-r\right)=x_{7}-x_{C_{2}}-r .
\end{array}\right.
$$

The theoretical interfaces positions determined by $\phi(x)=0$ lie at $x_{\Gamma_{1}}=x_{C_{1}}-r, x_{\Gamma_{2}}=x_{C_{1}}+r$, $x_{\Gamma_{3}}=x_{C_{2}}-r$ and $x_{\Gamma_{4}}=x_{C_{2}}+r$.

Next, let us compute the linearly approximated position of the interface $\Gamma_{1}$ with the aid of the relations (11) and (12):

$$
\widetilde{x}_{\Gamma_{1}}=x_{1}+\xi\left(x_{2}-x_{1}\right) \text { with } \xi=-\frac{\phi^{1}}{\phi^{2}-\phi^{1}} .
$$

Substituting the expressions of $\phi^{1}$ and $\phi^{2}$ into (13) yields

$$
\widetilde{x}_{\Gamma_{1}}=x_{C_{1}}-r \equiv x_{\Gamma_{1}} .
$$

So, the approximated location of $\Gamma_{1}$ turns out to be exact. Similarly, we can show that the linear approximations of the positions of $\Gamma_{3}$ and $\Gamma_{4}$ are also exact. In contrast, we have

$$
\widetilde{x}_{\Gamma_{2}}=x_{3}-\frac{x_{3}-x_{C_{1}}-r}{x_{C_{2}}+x_{C_{1}}-x_{3}-x_{4}}\left(x_{3}-x_{4}\right) \neq x_{\Gamma_{2}} .
$$

It is worth noting that even in this 1-D case where the interfaces positions would be expected to be exactly reproduced, some interfaces suffer a position shift. This phenomenon is illustrated in two dimensions in figure $5 \mathrm{~b}$ ). For a complex microstructure with a high volume fraction of inclusions, when the element size is not sufficiently small in comparison with the distance between two inclusions, the resulting interface position shift artefact may induce a significant error in calculating the inclusion volume fraction, which constitutes an additional source of error in numerically evaluating effective properties. 


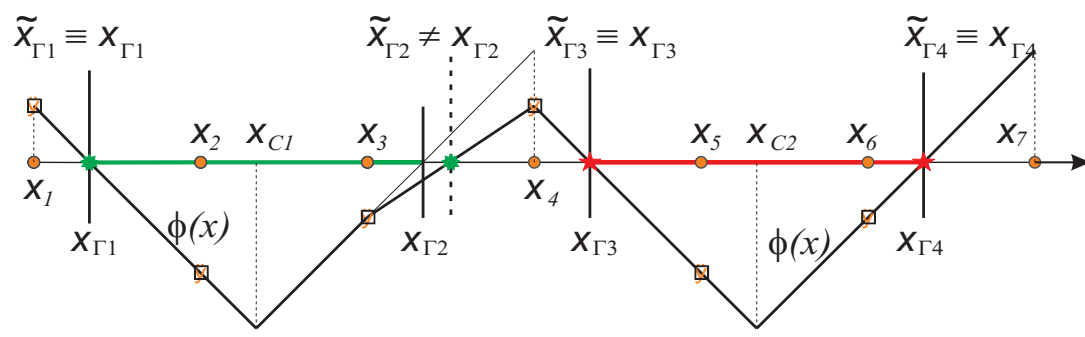

n Nodal values of $\phi(x)$

$\star$ Coordinates of the approximated interface positions

- Nodes of the elements

a)

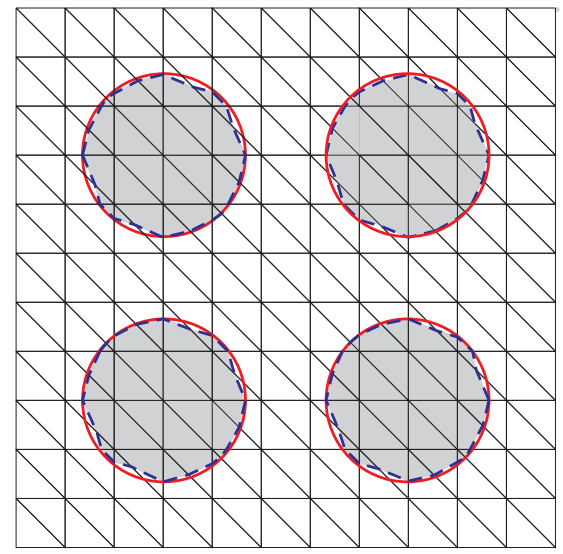

b)

Artificial interface distortion

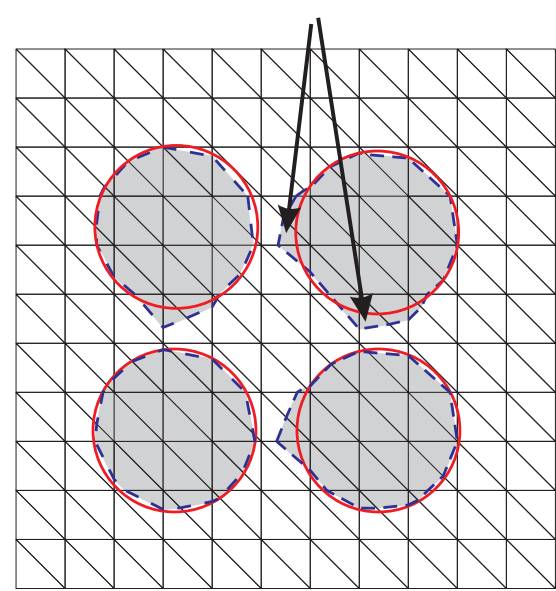

c)

Figure 5. a) Level-set function of two nearby 1D-inclusions, associated with negative values of $\phi(x)$. Nodes of 1D elements are indicated by circles; b) Theoretical (red solid lines) interfaces of several circular inclusions and linear approximation (dotted lines) using a single level-set. Inclusions are separated by one row of elements; c) Theoretical (red solid lines) interfaces of several circular inclusions and linear approximation (dotted lines) using a single level-set. Artificial distortion of interface approximation when two connected elements are each cut by one interface.

\subsection{Enrichment deficiency}

The aforementioned two artefacts being related to the level-set method, we now show an artefact due to the enriched approximation scheme. Indeed, when the support of an element is cut by more than one interface, the enriched approximation in XFEM using a single additional d.o.f. for each spatial field component is not rich enough to approximate a piece-wise linear field in the elements connected to the node. To show this, we consider a one-dimensional example as illustrated in figure 6 . A bar is discretized by two 1D linear elements. The material forming the bar is piece-wise homogeneous, and the interfaces between the phases are located at points $M$ and $N$. A level-set function $\phi(x)$ is defined such as $\phi(x)>0$ for $x$ in phase $1, \phi(x)<0$ 


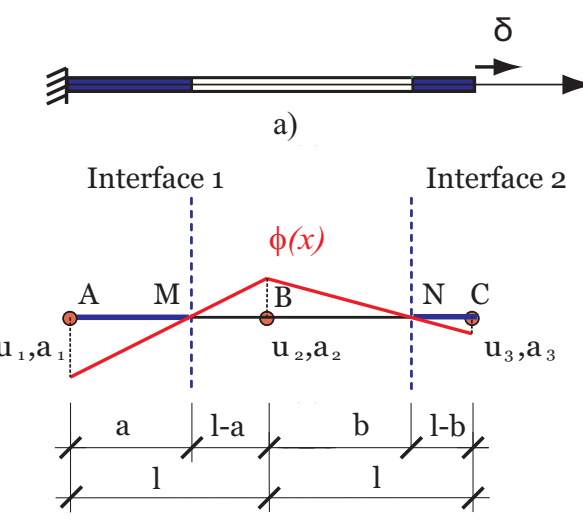

b)

Figure 6. a) Problem of piece-wise homogeneous bar, b) Level-set function and additional d.o.f. of two $1 \mathrm{D}$ linear elements cut by two interfaces within XFEM.

for $x$ in phase 2 and $\phi(x)=0$ for $x$ at an interface. In order to avoid the aforementioned two artefacts, we construct an ad-hoc level-set function defined by

$$
\phi^{1}=-\frac{c a}{l-a}, \quad \phi_{2}=c, \quad \phi^{3}=-\frac{c(l-b)}{b} .
$$

Above, $a$ and $b$ are two lengths strictly smaller than the length $l$ of an element and $c>0$ is a constant.

Let us define the coordinates of points $A, M, B, N$ and $C$ of the bar by $x_{A}, x_{M}, x_{B}$, $x_{N}$ and $x_{C}$. Small strains are assumed, and phases 1 et 2 are taken to be linearly elastic and characterized by Young's moduli $E_{1}$ and $E_{2}$, respectively. The bar is clamped at $x_{A}$ and subjected to a displacement $\delta$ at $x_{C}$.

Applying the approximation (1) to the elements $\left\{x_{A}-x_{B}\right\}$ and $\left\{x_{B}-x_{C}\right\}$, we obtain

$$
\begin{array}{ll}
u^{h}(x)=u_{1} N_{1}(x)+u_{2} N_{2}(x)+\left[a_{1} N_{1}(x)+a_{2} N_{2}(x)\right] \psi(x), & x \in\left[x_{A}, x_{B}\right], \\
u^{h}(x)=u_{2} N_{2}(x)+u_{3} N_{3}(\mathbf{x})+\left[a_{2} N_{2}(x)+a_{3} N_{3}(x)\right] \psi(x), & x \in\left[x_{B}, x_{C}\right],
\end{array}
$$

where $N_{i}(x)$ are 1D linear finite element shape functions. Concerning the boundary conditions, we have $u_{1}=0$ and $u_{3}=\delta$. We first compute the energies for the segments $\left\{x_{A}-x_{M}\right\}$, $\left\{x_{M}-x_{B}\right\},\left\{x_{B}-x_{N}\right\}$ and $\left\{x_{N}-x_{C}\right\}$ as

$$
W_{x_{i}-x_{j}}=\int_{x_{i}}^{x_{j}} \frac{1}{2} E_{i}\left(\frac{d u^{h}(x)}{d x}\right)^{2} d x .
$$

We then minimize the total energy with respect to the unkowns $u_{2}, a_{1}, a_{2}$ and $a_{3}$. Choosing the numerical values $a=0.5 \mathrm{~m}, b=0.5 \mathrm{~m}$ and $l=1 \mathrm{~m}$, it follows that 


$$
\begin{aligned}
& a_{1}=-\delta \frac{3\left(E_{1}-E_{2}\right)}{8 c\left(E_{1}+E_{2}\right)}, \\
& a_{2}=0, \\
& a_{3}=\delta \frac{3\left(E_{1}-E_{2}\right)}{8 c\left(E_{1}+E_{2}\right)}, \\
& u_{2}=\delta / 2 .
\end{aligned}
$$

Introducing these expressions into the finite element formulae for the displacement and energy yields the expressions

$$
\begin{aligned}
u_{A B}(x)= & \frac{-\delta\left(-7 E_{1} x-E_{2} x+3 E_{1} x|2 x-1|+3 E_{1}\right)}{8\left(E_{1}+E_{2}\right)} \\
& +\frac{-\delta\left(-3 E_{1}|2 x-1|-3 E_{2} x|2 x-1|-3 E_{2}+3 E_{2}|2 x-1|\right)}{8\left(E_{1}+E_{2}\right)}, \\
u_{B C}(x)= & \frac{-\delta\left(-7 E_{1} x-E_{2} x+3 E_{1} x|2 x-3|+3 E_{1}\right)}{8\left(E_{1}+E_{2}\right)} \\
& +\frac{-\delta\left(-3 E_{1}|2 x-3|-3 E_{2} x|2 x-3|-3 E_{2}+3 E_{2}|2 x-3|\right)}{8\left(E_{1}+E_{2}\right)}, \\
W_{\text {total }}= & \delta^{2} \frac{E_{1}^{2}+14 E_{1} E_{2}+E_{2}^{2}}{32\left(E_{1}+E_{2}\right)},
\end{aligned}
$$

where the displacement expressions $u_{A B}$ and $u_{B C}$ are valid for $x \in\left[x_{A}, x_{B}\right]$ and $x \in\left[x_{B}, x_{C}\right]$, respectively, and $W_{\text {total }}$ stands for the approximated total energy of the bar.

It is easy to analytically obtain the exact energy of the bar:

$$
W_{\text {total }}^{\text {exact }}=\delta^{2} \frac{E_{1} E_{2}}{2\left(E_{1}+E_{2}\right)} .
$$

From equations (24) and (25), we can calculate the relative energy error as

$$
\Delta W^{X F E M}=\frac{W_{\text {total }}^{\text {exact }}-W_{\text {total }}}{W_{\text {total }}^{\text {exact }}}=\frac{\left(E_{1}-E_{2}\right)^{2}}{16 E_{2} E_{1}} .
$$

The displacement and strain distributions are plotted for the numerical values $\delta=0.05 \mathrm{~m}$, $E_{1}=10 \mathrm{MPa}$ and $E_{2}=100 \mathrm{MPa}$ in figure 7 . It is seen that the Level-set/XFEM approximation cannot reproduce exactly the piece-wise linear exact solution, so that an enrichment deficiency takes place. For a complex microstructure with a high volume fraction of inclusions, when the mesh size is not small enough compared with the distance between inclusions, the enrichment deficiency just shown constitutes a source of error in addition to the two previous ones.

\section{MULTIPLE LEVEL-SETS/XFEM METHOD ( $\mu$-XFEM)}

\section{1. $\mu-X F E M$ approach}

To avoid the aforementioned actefacts, we now propose an improved version of the XFEM/level-set method, called "multiple Level-Set XFEM" ( $\mu$-XFEM). Precisely, in the 

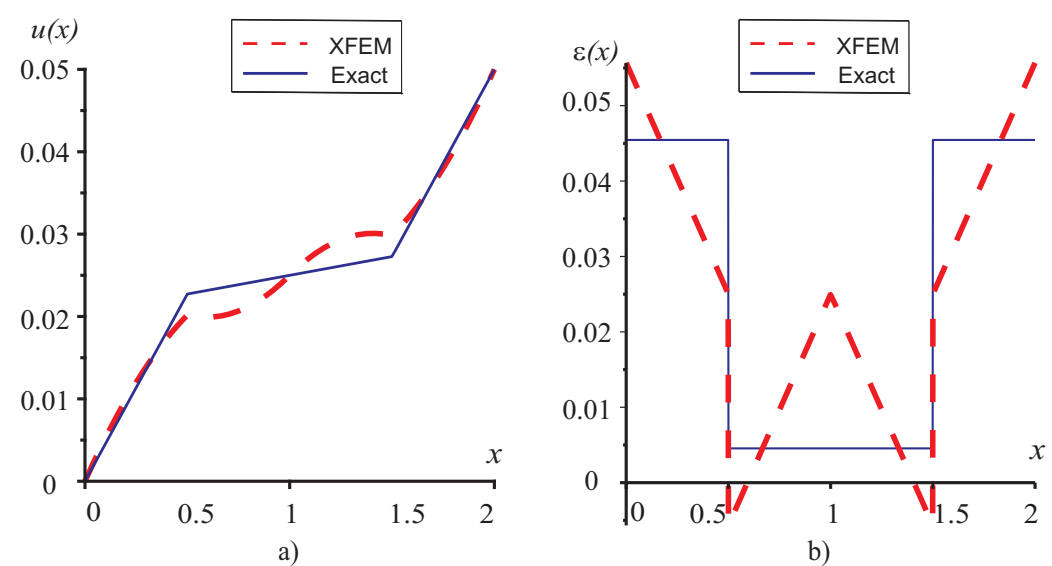

Figure 7. Comparison between the exact and XFEM/level-set solutions for the 1D problem with two interfaces: a) displacements and b) strains.

enriched approximation, each interface is endowed with an individual enrichment, so that the displacement approximation takes the form

$$
\mathbf{u}^{h}(\mathbf{x})=\sum_{i \in \mathcal{S}} N_{i}(\mathbf{x}) \mathbf{u}_{i}+\sum_{k}^{N_{\text {int }}} \sum_{j \in \mathcal{S}^{e k}} N_{j}(\mathbf{x}) \psi^{k}\left(\phi^{k}(\mathbf{x})\right) \mathbf{a}_{j}^{k} .
$$

In the above equation, $\mathbf{a}_{j}^{k}$ are nodal unknowns, the nodal set $\mathcal{S}^{e k}$ is defined as

$$
\mathcal{S}^{e k}=\left\{j \mid j \in \mathcal{S}, \omega_{j} \cap \Gamma^{k} \neq 0\right\},
$$

and $\psi^{k}\left(\phi^{k}(\mathbf{x})\right)$ is an enrichment function (Eq. (10)) constructed via the level-set function $\phi^{k}$ of inclusion $k$ with boundary $\Gamma^{k}$. An illustration in the case of three interfaces cutting a linear triangular element is provided in figure 8 a). The general level-set function $\phi^{k}$ takes the form

$$
\Gamma_{k}=\left\{\mathbf{x} \in \mathbb{R}^{d} \mid \phi^{k}(\mathbf{x})=0\right\} .
$$

We can opt to choose the signed distance function for $\phi^{k}(\mathbf{x})$, which is given by Eq. (4). For a spherical inclusion $k, \phi^{k}(\mathbf{x})$ is expressed by

$$
\phi^{k}(\mathbf{x})=\left\{\left\|\mathbf{x}-\mathbf{x}_{c}^{k}\right\|-r^{k}\right\} .
$$

The enriched approximation (26) allows us to treat several discontinuities present in a single element, as depicted schematically in figure $8 \mathrm{~b}$ ). It also removes all the numerical artefacts for nearby inclusions, as shown in the following. However, this technique induces several difficulties: a) the storage of the level-set functions in the case of a high number of inclusions; b) the numerical integration over an element cut by more than one interface; c) the assembly of the stiffness matrix. We propose simple solutions to avoid these difficulties in the next section. 


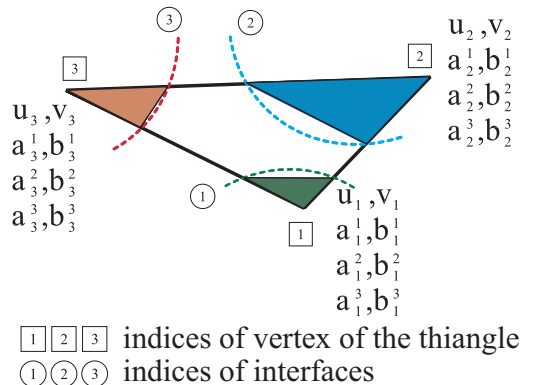

(1) (2) (3) indices of interfaces

a)

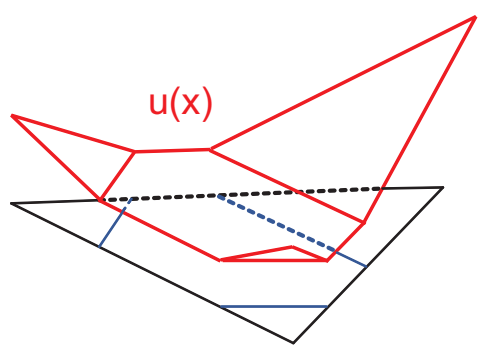

b)

Figure 8. a) Additional d.o.f. for a 2D element cut by three interfaces; $u_{i}$ and $v_{i}$ denote classical FEM d.o.f., while $a_{i}^{k}$ and $b_{i}^{k}$ denote enrichment d.o.f. related to interfaces $k$; b) approximated discontinuous displacement field in an element cut by three interface

\subsection{Level-sets storage}

When the domain contains a large number of inclusions, storing each level-set function $\phi^{k}$ (defined at each node of the domain) requires very large memory capacities. It can be simplified by storing the values of level-set function $\phi_{k}$ of interface $\Gamma_{k}$ only at nodes whose support is cut by $\Gamma_{k}$. In this situation, a sparse storage strategy may be used for reducing the memory requiring.

An example of practical storage strategy is to define two sparse matrices LSnodes and LSelements, where $\operatorname{LSnodes}(j, k)=\phi_{k}\left(x_{j}\right)$ if the support of node $x_{j}$ is cut by the interface $\Gamma_{k}$ and the line $i$ of LSelements contains the indices of the interfaces cut by the element $i$.

\subsection{Assembly of the stiffness matrix}

Within XFEM, each node whose support is cut by an interface is associated with $D \times 2$ d.o.f. However, in $\mu$-XFEM, each node whose support is cut by $N_{\text {inter }}$ interfaces is associated with $D \times\left(1+N_{\text {inter }}\right)$ d.o.f.. When connected elements do not contain the same number of cutting interfaces, the assembly operation is not trivial. We propose a technique to overcome this difficulty. For the sake of simplicity, we restrict the discussion to the $2 \mathrm{D}$ case $(\mathrm{D}=2)$. Let us consider a node $i$ with two displacement components $u_{i}, v_{i}$ and $n_{i}$ additional d.o.f. $a_{i}^{1}, a_{i}^{2}, . ., a_{i}^{D n_{i}}$. The number $n_{i}$ is the number of non-zero indices in the $i-t h$ line of $\mathbf{L V}$ inter array defined in section 4.2. One choice for storing the degrees of freedom in the global vector of unknowns $\mathbf{d}$ in (41) is as follows. indices of nodal displacements are $2 i-1$, $2 i$, while the additional d.o.f. indices are $\mathcal{A}(i)+1$ to $\mathcal{A}(i)+D n_{i}$, where the vector $\mathcal{A}$ is defined as

$$
\mathcal{A}(i)=D\left[N+\sum_{j=1}^{i-1} \mathcal{L}(\text { LVinter }(j))\right],
$$

with $N$ being the total number of nodes in the mesh and $\mathcal{L}($.$) is an operator defining the$ number of non-zero values in vector (.). 


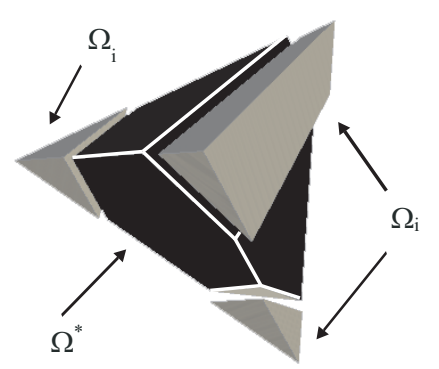

Figure 9. A tetrahedral element cut by three interfaces and partitioning of the element domain into subdomains $\Omega_{i}$ and $\Omega^{*}$.

\subsection{Numerical integration}

In the XFEM/level-set method, if an element is cut by an interface, the element must be decomposed into subdomains for numerical integration purpose. In the case of linear elements cut by one interface, the different geometrical cases are restricted [5]. However, when an element is cut by more than one interface, domain with complex polyhedral shapes can occur, leading to non trivial geometrical partitioning. This issue is illustrated in figure 9. To avoid this difficulty, we propose a simple strategy. The idea is to decompose the integration such as treating only simple intersection problems: if we consider the case in figure 9, integration is easily carried out in subdomains $\Omega_{i}$ using a Gauss quadrature rule. The difficulty is associated with the polyhedral domain $\Omega^{*}=\Omega \backslash\left(\bigcup_{i} \Omega_{i}\right)$, which shape depends on the number of interfaces cutting the element and the number of intersection points between each vertex of the element.

We aim here to evaluate the integration over tetrahedral domain $\Omega$ of function $f: \Omega \subset$ $\mathbb{R}^{3} \rightarrow \mathbb{R}$ given as follows:

$$
f(\mathbf{x})=\left\{\begin{array}{ll}
f^{*}(\mathbf{x}) & \text { if } \mathbf{x} \in \Omega^{*} \\
f_{i}(\mathbf{x}) & \text { if } \mathbf{x} \in \Omega_{i}
\end{array} .\right.
$$

where the function $f_{i}(\mathbf{x})$ and $f^{*}(\mathbf{x})$ are associated with the inclusions and matrix materials, respectively. We can decompose this integration into

$$
\int_{\Omega} f(\mathbf{x}) d \Omega=\sum_{i} \int_{\Omega_{i}} f_{i}(\mathbf{x}) d \Omega+\int_{\Omega^{*}} f^{*}(\mathbf{x}) d \Omega .
$$

To overcome numerical difficulties related to the integration of $f^{*}(\mathbf{x})$ over the domain $\Omega^{*}$, we use a simple superposition technique as follows:

$$
\int_{\Omega^{*}} f^{*}(\mathbf{x}) d \Omega=\int_{\Omega} f^{*}(\mathbf{x}) d \Omega-\sum_{i}\left(\int_{\Omega_{i}} f^{*}(\mathbf{x}) d \Omega\right),
$$

where $f^{*}: \Omega \subset \mathbb{R}^{d} \rightarrow \mathbb{R}$ is extended over the domain $\Omega$.

By introducing the above formula into Eq. (32), the integration of $f(x)$ over the tetrahedral 


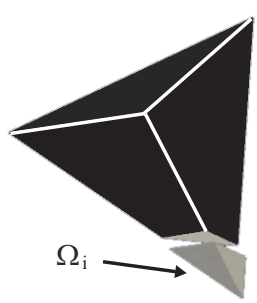

a)

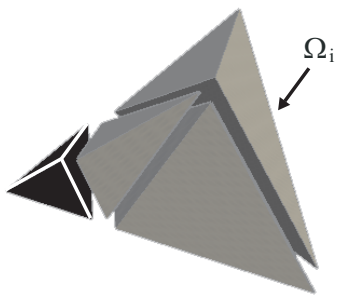

b)

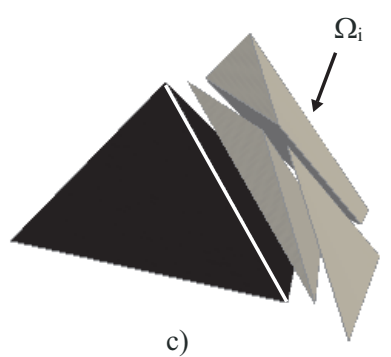

c)

Figure 10. Three possibilities for subdivision of subdomains $\Omega_{i}$ into tetrahedra.

domain is expressed as:

$$
\int_{\Omega} f(\mathbf{x}) d \Omega=\int_{\Omega} f^{*}(\mathbf{x}) d \Omega+\sum_{i}\left(\int_{\Omega_{i}}\left(f_{i}(\mathbf{x})-f^{*}(\mathbf{x})\right) d \Omega\right) .
$$

Both right-hand terms in Eq. (34) can be easily calculated, as $\Omega_{i}$ are composed of only a few tetrahedra. Then it only requires: (i) constructing intersections between the element $\Omega^{e}$ and the linear approximations of level-set functions $\phi^{k}$; (ii) partitioning the domains $\Omega_{i}$ into tetrahedra. The intersection points $\mathbf{x}_{p}$ are given by:

$$
\mathbf{x}_{p}=\mathbf{x}_{i}+\xi\left(\mathbf{x}_{j}-\mathbf{x}_{i}\right), \quad \xi=-\frac{\phi_{i}^{k}}{\phi_{j}^{k}-\phi_{i}^{k}},
$$

where $\mathbf{x}_{j}$ are two edge points of an element, $\phi_{i}^{k}$ and $\phi_{j}^{k}$ are values of the level-set function of interface $\Gamma_{k}$ at the points $\mathbf{x}_{i}$, and $\mathbf{x}_{j}$. We note that here an edge is cut by the interface $\Gamma_{k}$ if and only if $\phi_{i}^{k} \phi_{j}^{k}<0$. In this framework, each subdomain can easily be associated with different material properties according to the sign of $\phi_{i}^{k}(\mathbf{x})$. Once the domains $\Omega_{i}$ are constructed, the subdivision of $\Omega_{i}$ into tetrahedra is easily obtained as explained in [5] by looping over the interfaces cutting the tetrahedral domain $\Omega$. The possibilities for the subdivision is illustrated in the figure 10. The black tetrahedra belong to matrix and the gray ones to the inclusions. The subdivision must be done in the gray subdomain. The figures $10 \mathrm{a}$ ) and b) correspond to the case where the interface cuts three edges of element and the figure $10 \mathrm{c}$ ) shows the interface cutting four edges of the element.

\subsection{Governing equations}

In this section, we present the governing equations of linear elastostatics, together with the weak form and associated discrete system for $\mu$-XFEM.

4.5.1. Strong form We consider a body which is described by an open bounded domain $\Omega \subset \mathbb{R}^{d}$, with boundary $\partial \Omega$. The latter is composed of two disjoint complementary parts $\partial \Omega_{u}$ and $\partial \Omega_{t}$, where the Dirichlet (displacement) and Neumann (tractions) boundary conditions are prescribed, respectively. The field equations of elastostatics are: 


$$
\begin{gathered}
\nabla \cdot \boldsymbol{\sigma}+\mathbf{b}=0 \text { in } \Omega, \\
\boldsymbol{\sigma}=\mathbb{C}: \boldsymbol{\varepsilon},
\end{gathered}
$$

where $\boldsymbol{\varepsilon}=\left(\nabla \mathbf{u}+\nabla \mathbf{u}^{T}\right) / 2$ and $\mathbb{C}$ is the fourth-order tensor of elastic moduli. The essential and natural boundary conditions are

$$
\begin{gathered}
\mathbf{u}=\overline{\mathbf{u}} \text { on } \partial \Omega_{u}, \\
\boldsymbol{\sigma} \mathbf{n}=\overline{\mathbf{t}} \text { on } \partial \Omega_{t},
\end{gathered}
$$

where $\mathbf{n}$ is the unit outward normal to $\partial \Omega$.

4.5.2. Weak form and discrete system The weak form associated with Eqs. (36)-(39) is given by finding $\mathbf{u} \in \mathcal{D}=\left\{\mathbf{u} \mid \mathbf{u}=\overline{\mathbf{u}}\right.$ on $\left.\partial \Omega_{\mathrm{u}}, \mathbf{u} \in \mathrm{H}^{1}(\Omega)\right\}$ such that

$$
\int_{\Omega} \sigma: \varepsilon(\delta \mathbf{u}) d \Omega=\int_{\partial \Omega_{t}} \overline{\mathbf{t}} \cdot \delta \mathbf{u} d \Gamma+\int_{\Omega} \delta \mathbf{u} \cdot \mathbf{b} d \Omega \quad \forall \delta \mathbf{u} \in H_{0}^{1}(\Omega),
$$

where $H_{0}^{1}(\Omega)=\left\{\delta \mathbf{u} \mid \delta \mathbf{u} \in H^{1}(\Omega), \delta \mathbf{u}=0\right.$ on $\left.\partial \Omega_{\mathrm{u}}\right\}$

By substituting the displacement field defined in (26) into the weak form (40) we obtain the discrete system of linear ordinary equations:

$$
\mathbf{K d}=\mathbf{f}, \quad \mathbf{d}=\left\{\begin{array}{ll}
\mathbf{u} & \mathbf{a}
\end{array}\right\}^{\mathrm{T}},
$$

where $\mathbf{d}$ and $\mathbf{a}$ are nodal unknowns and $\mathbf{K}$ and $\mathbf{f}$ are the global stiffness matrix and external force vector, respectively. More precisely, the matrix $\mathbf{K}$ and vector $\mathbf{f}$ are defined by

$$
\mathbf{K}=\int_{\Omega} \mathbf{B}^{T} \mathbf{C B} d \Omega, \quad \mathbf{f}=\int_{\Gamma_{\mathrm{t}}} \mathbf{N}^{\mathrm{T}} \overline{\mathbf{t}} \mathrm{d} \Gamma+\int_{\Omega} \mathbf{N}^{\mathrm{T}} \mathbf{b} \mathrm{d} \Omega,
$$

where $\mathbf{B}$ and $\mathbf{N}$ are the matrices of shape function derivatives and shape functions associated with the approximation scheme (26), and $\mathbf{C}$ is the matrix corresponding to the elasticity tensor $\mathbb{C}$.

\section{TREATMENT OF NUMERICAL ARTEFACTS WITHIN $\mu$-XFEM}

In this section, we show how the different numerical artefacts pointed out above can be avoided using $\mu-X F E M$.

\subsection{Artificial percolation}

In $\mu$-XFEM, each inclusion is associated with a single level-set function, as shown in figure 11. Then the percolation artefact no longer occurs. To illustrate this, let us consider the simple one-dimensional example depicted in figure $12 \mathrm{a}$ ). The interface $\Gamma_{1}$ is related to the level-set function $\phi^{1}(\mathbf{x})$ while a second level-set function $\phi^{2}(\mathbf{x})$ is used to described $\Gamma_{2}$. Unlike the case 

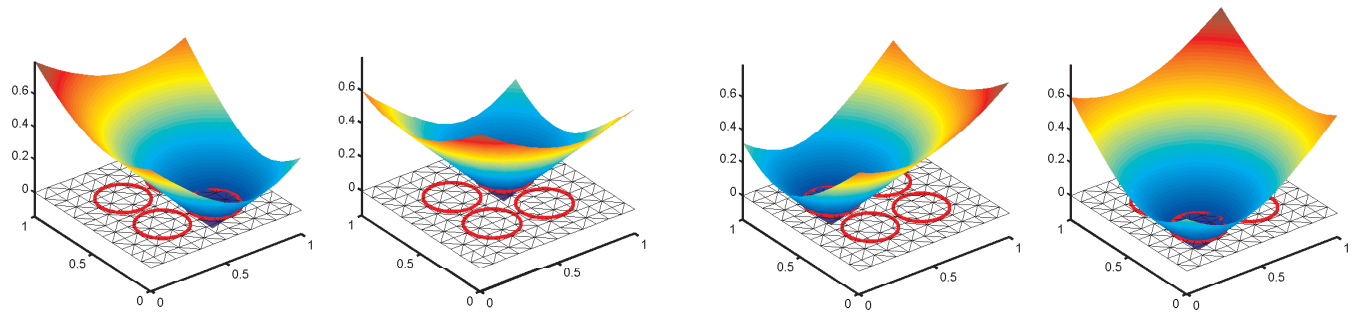

Figure 11. Description of four interfaces within $\mu$-XFEM: each inclusion is associated with a single level-set function.

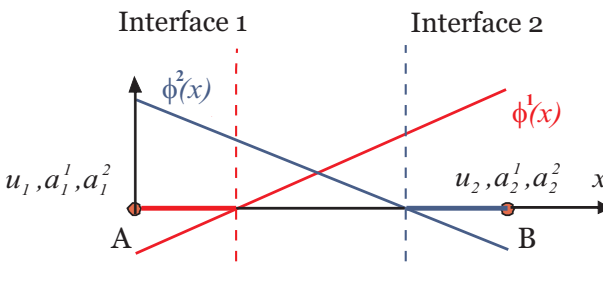

a)

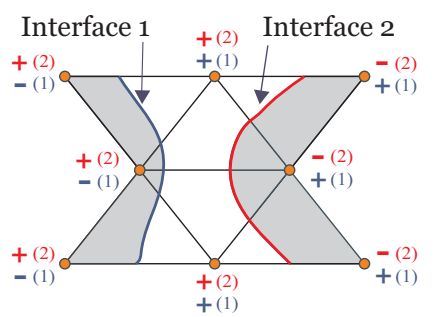

b)

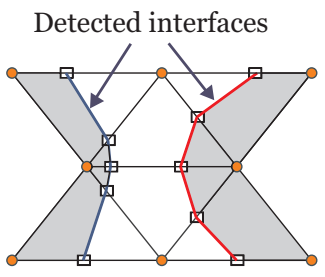

c)

Figure 12. A single element cut by two interfaces within $\mu$-XFEM; each inclusion is associated with one level-set function; b) theoretical interfaces of two nearby inclusions and background mesh. The $"+(\mathrm{k}) "$ and "-(k)" indicate the sign of the $\phi^{k}$ level-set nodal values; c) Linear approximation of the zero-level-sets.

when a single level-set was used in section 3.1, figure 4 b) and $4 \mathrm{c}$ ), both interfaces are detected in a single element, and the percolation is removed.

A two-dimensional example is described in figures $12 \mathrm{~b}$ ) and $12 \mathrm{c}$ ). The nodal signs values of the level-set functions $\phi^{1}(x)$ and $\phi^{2}(x)$ related to the interfaces 1 and 2 are indicated. Using (35), intersections between the mesh and the linear approximation of the level-set functions are indicated by square dots. It can be observed that, in contrast to the case of using a single level-set function (figure $4 \mathrm{c}$ ), there is no bridge between the inclusions.

\subsection{Artificial interface distortion}

The artefact related to interface detection using a single level-set function is trivially removed when employing several level-set functions. As an illustration, we consider the one-dimensional example of figure 13: two connected elements each contain an interface. When appliying $\mu$ XFEM, the interfaces 1 and 2 are respectively deduced from the nodal values of the level-set functions $\phi^{1}(x)$ and $\phi^{2}(x)$. Each interface detection is equivalent to the case of a single interface using a level-set function, so no additional interface distortion is produced. 


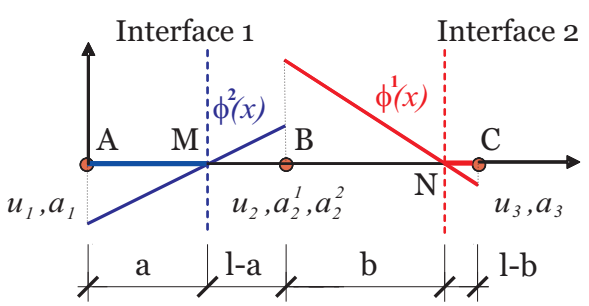

Figure 13. Additional d.o.f. in two elements cut by two interfaces within $\mu$-XFEM.

\subsection{Enrichment deficiency}

As new d.o.f. are introduced within the $\mu$-XFEM, it is expected that the artefact highlighted in section 3.3 will be removed. We consider the same problem studied in section 3.3 and show that the exact solution is reproduced when applying $\mu$-XFEM. The nodal values of level-set functions $\phi^{1}(x)$ and $\phi^{2}(x)$ (see figure 13) defined in (29) are determined as follows:

$$
\phi_{1}^{1}=-a ; \quad \phi_{2}^{1}=1-\mathrm{a} ; \quad \phi_{2}^{2}=\mathrm{b} ; \quad \phi_{3}^{2}=\mathrm{b}-1 .
$$

Applying the approximation (26) to the elements $\left\{x_{A}-x_{B}\right\}$ and $\left\{x_{B}-x_{C}\right\}$, we obtain

$$
\begin{aligned}
& u^{h}(\mathbf{x})=u_{1} N_{1}(\mathbf{x})+u_{2} N_{2}(\mathbf{x})+\left[a_{1}^{1} N_{1}(\mathbf{x})+a_{2}^{1} N_{2}(\mathbf{x})\right] \psi^{1}(\mathbf{x}) \quad \forall x \in\left\{x_{A}, x_{B}\right\}, \\
& u^{h}(\mathbf{x})=u_{2} N_{2}(\mathbf{x})+u_{3} N_{3}(\mathbf{x})+\left[a_{2}^{2} N_{2}(\mathbf{x})+a_{3}^{2} N_{3}(\mathbf{x})\right] \psi^{2}(\mathbf{x}) \quad \forall x \in\left\{x_{B}, x_{C}\right\},
\end{aligned}
$$

where $\psi^{k}(\mathbf{x})$ are the enrichment functions defined by

$$
\begin{aligned}
& \psi^{1}(\mathbf{x})=\left|\phi_{1}^{1}\right| N_{1}(\mathbf{x})+\left|\phi_{2}^{1}\right| N_{2}(\mathbf{x})-\left|\phi_{1}^{1} N_{1}(\mathbf{x})+\phi_{2}^{1} N_{2}(\mathbf{x})\right|, \\
& \psi^{2}(\mathbf{x})=\left|\phi_{2}^{2}\right| N_{2}(\mathbf{x})+\left|\phi_{3}^{2}\right| N_{3}(\mathbf{x})-\left|\phi_{2}^{2} N_{2}(\mathbf{x})+\phi_{3}^{2} N_{3}(\mathbf{x})\right| .
\end{aligned}
$$

As in section 3.3, we apply the boundary conditions $u_{1}=0$ and $u_{3}=\delta$ and minimize the total energy with respect to the unknowns of the problem. Here, the free parameters are $u_{2}, a_{1}^{1}, a_{2}^{1}$, $a_{2}^{2}$ and $a_{3}^{2}$. Using $a=2 / 3 \mathrm{~m}, b=2 / 3 \mathrm{~m}, l=1 \mathrm{~m}$, we obtain:

$$
\begin{aligned}
& a_{1}=-\frac{1}{2} \frac{\left(E_{1}-E_{2}\right) \delta}{E_{1}+E_{2}}, \\
& a_{2}^{1}=-\frac{1}{2} \frac{\left(E_{1}-E_{2}\right) \delta}{E_{1}+E_{2}}, \\
& a_{2}^{2}=\frac{1}{2} \frac{\left(E_{1}-E_{2}\right) \delta}{E_{1}+E_{2}}, \\
& a_{3}^{2}=\frac{1}{2} \frac{\left(E_{1}-E_{2}\right) \delta}{E_{1}+E_{2}}, \\
& u_{2}=\frac{1}{3} \frac{\left(E_{1}+2 E_{2}\right) \delta}{E_{1}+E_{2}} .
\end{aligned}
$$

Introducing these values in the displacement fields and total energy expressions yields: 


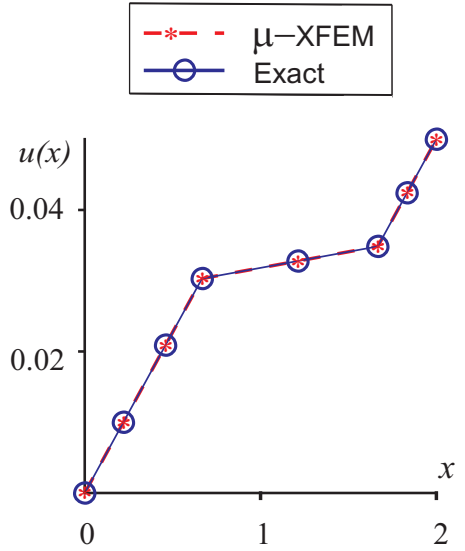

a)

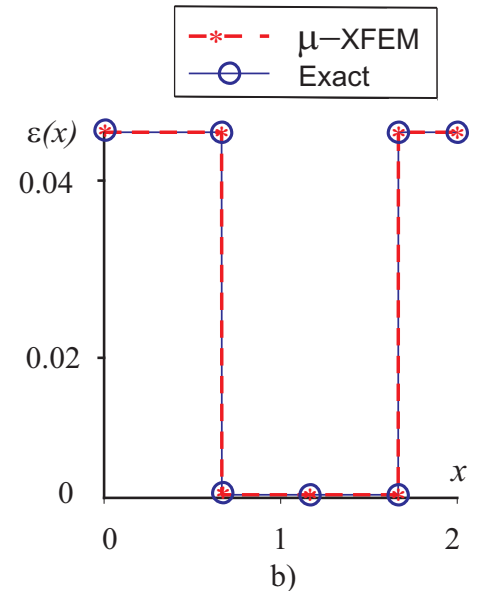

b)

Figure 14. Comparison between exact and $\mu$-XFEM/level-set solutions for the $1 \mathrm{D}$ problem with two interfaces: a) displacements and b) strains.

$$
\begin{aligned}
u_{A B}^{\mu-X F E M}(x) & =\frac{1}{6} \frac{\left(3 E_{1} x+3 E_{2} x-2 E_{1}+E_{1}|3 x-2|+2 E_{2}-E_{2}|3 x-2|\right) \delta}{E_{1}+E_{2}}, \\
u_{B C}^{\mu-X F E M}(x) & =-\frac{1}{6} \frac{\left(-3 E_{1} x-3 E_{2} x-E_{1}+E_{1}|3 x-5|+E_{2}-E_{2}|3 x-5|\right) \delta}{E_{1}+E_{2}}, \\
W_{\text {total }} & =\delta^{2} \frac{E_{1} E_{2}}{2\left(E_{1}+E_{2}\right)} .
\end{aligned}
$$

Using the expression of the exact total energy (25) we obtain the relative energy error

$$
\Delta W^{\mu-X F E M}=\frac{W_{\text {total }}^{\text {exact }}-W_{\text {total }}}{W_{\text {total }}^{\text {exact }}}=0 .
$$

We show in figure 14 the displacement and strain along $x$, using the numerical parameters $\delta=0.05 \mathrm{~m}, E_{1}=10 \mathrm{MPa}$ and $E_{2}=100 \mathrm{MPa}$. The exact solutions are reached within machining precision.

\subsection{Remarks}

(i) Note that the different artefacts hold only when a linear approximation is used for the zero level-set approximation. Higher-order approximation scheme (e.g. quadratic elements) may be used instead of the present method. However, the proposed technique aims at keeping the simplicity of the linear method for numerical integration and interface approximation.

(ii) When the distance between two inclusions is of the order of the mesh size, the $\mu$ XFEM yields exact solution for piece-wise linear functions, while XFEM induces errors. When the mesh is fine enough, the numerical results given by both methods are indistinguishable. 


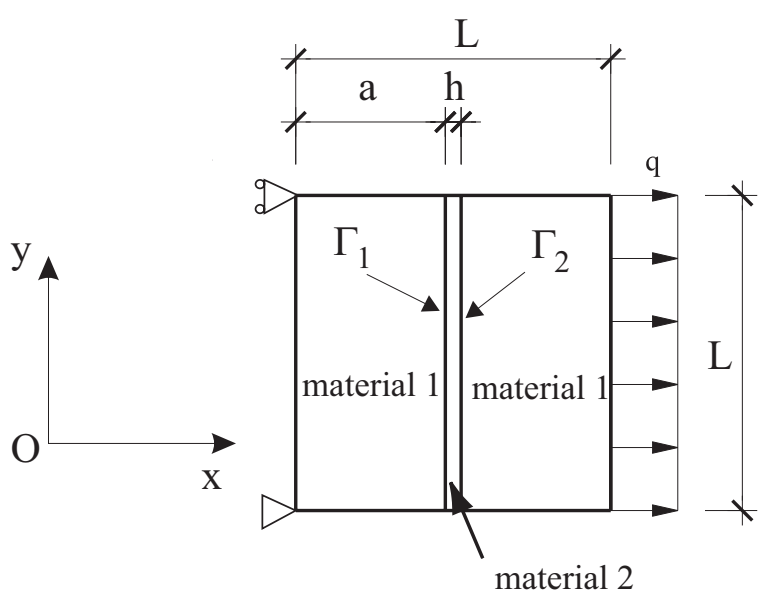

Figure 15. Geometry of the problem and boundary conditions.

(iii) Approaches combining XFEM with mesh refinement have been proposed in [8]. It is worth noting that in some cases when inclusions are close to each other, this kind of technique might be useful to accurately describe the stress fields between two inclusions. When microstructures are very complex as shown in the example of section 6.4.2, a mesh refinement strategy would probably lead to a uniform refined mesh in the whole domain. Nevertheless, for simpler cases the present method and mesh refinement are fully compatible and could lead to an even more efficient XFEM strategy for describing complex microstructures.

(iv) In the proposed approach, a different material can be assigned to each phase, which is difficult using a single level-set function.

\section{NUMERICAL EXAMPLES}

\subsection{Interphase patch test}

In this first example, we propose a patch test where a thin interphase is located between two phases. The geometry of the problem is depicted in figure 15. The aim of this benchmark is to show that the proposed method allows reproducing a piece-wise linear solution in the presence of nearby interfaces, while the XFEM/level-set fails in this case.

The square domain is clamped on one side and subjected to a homogeneous force distribution $\mathbf{q}=q \mathbf{e}_{x}$ on its opposite side. The exact solution of this problem reads

$$
u^{e x}(x)=\left\{\begin{array}{cc}
\frac{q}{E_{1}} x & 0 \leq x \leq a \\
\frac{q a}{E 1}+\frac{q}{E_{2}}(x-a) & a \leq x \leq a+h \\
\frac{q a}{E_{1}}+\frac{q h}{E_{2}}+\frac{q}{E_{1}}(x-a-h) & a+h \leq x \leq L
\end{array}\right.
$$

For the XFEM/Level-set method, the single level-set function $\phi(x)=\min \left(\phi^{1}(x), \phi^{2}(x)\right)$ with $\phi^{1}(x)=x-a$ and $\phi^{2}(x)=a+h-x$ is used. For $\mu-X F E M$, two level-set functions 


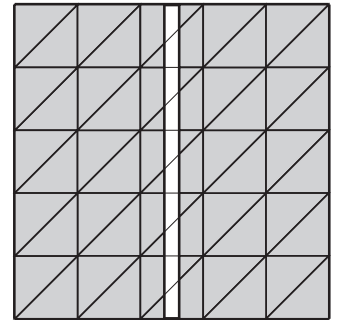

a)

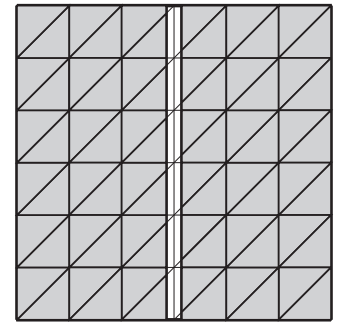

b)

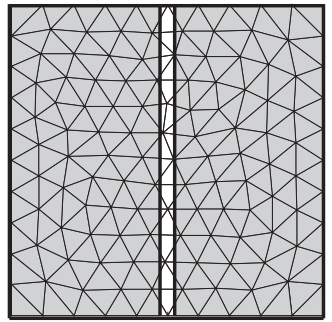

c)

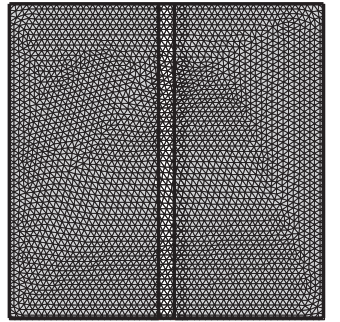

d)

Figure 16. a) Mesh with $\mathrm{n}=6$ (even). b) Mesh with $\mathrm{n}=7$ (odd). c) and d) unstructured meshes.

$\phi^{1}(x)$ and $\phi^{2}(x)$ (the same as defined for the single level-set) are respectively associated to the two interfaces $\Gamma_{1}$ and $\Gamma_{2}$. The following numerical parameters are adopted: $E_{1}=1 \mathrm{MPa}$, $\nu_{1}=0, E_{2}=100 \mathrm{MPa}, \nu_{2}=0, q=10 \mathrm{MPa} / \mathrm{m}, h=0.05 \mathrm{~m}$ and $a=0.45 \mathrm{~m}$. The indices 1 and 2 corresponding to the domains 1 and 2, respectively (see figure 15 a).

The RVE is meshed with linear triangles. We study two cases: (i) a regular mesh as illustrated in figures $16 \mathrm{a}$ ) and $16 \mathrm{~b}$ ); (ii) an unstructured mesh as shown in figures $16 \mathrm{c}$ ) and $16 \mathrm{~d}$ ). We compute the relative energy error norm defined by

$$
e=\frac{\left\|\mathbf{u}^{e x}-\mathbf{u}^{h}\right\|_{E}}{\left\|\mathbf{u}^{e x}\right\|_{E}}=\frac{\left(\int_{\Omega}\left(\varepsilon^{h}-\varepsilon^{e x}\right): \mathbf{C}:\left(\varepsilon^{h}-\varepsilon^{e x}\right) d \Omega\right)^{1 / 2}}{\left(\int_{\Omega} \varepsilon^{e x}: \mathbf{C}: \boldsymbol{\varepsilon}^{e x} d \Omega\right)^{1 / 2}}
$$

for increasing element densities associated with a characteristic size $h$. For the regular mesh, the results are depicted in figure 17. It can be noticed that using XFEM, an oscillating curve is obtained until a single element is contained within the layer. As expected, the XFEM can reproduce the solution roughly at the machine precision. The explanation for this oscillating behaviour comes when we distinguish two cases of meshes: the case when a node is contained within the layer (odd number of nodes in the $\mathrm{x}$-direction) and the case with an even number of nodes. The case of odd number of nodes corresponds to the artefact related to the lack of d.o.f., as highlighted in section 3.3. We have plotted separately the convergence curves for this case in figure 18 a). The case of an even number of nodes corresponds to the percolation artefact described in section 3.1. The convergence curve for this case is presented in figure 18 b).

In contrast, the $\mu$-XFEM can reproduce the solution exactly for any mesh. When the mesh is fine enough both XFEM and $\mu$-XFEM solutions coincide.

When an unstructured mesh is used, the different artefacts occur at the same time. We study the convergence of the solution for this case in figure 19, the value $h$ being defined by $h=\frac{1}{\sqrt{N}-1}$ with $N$ being the number of nodes in the mesh. Here again, the XFEM cannot reproduce the exact solution until a sufficient element density is reached to remove the different artefacts. In contrast, the $\mu$-XFEM can reproduce the solution at the machine precision for any mesh. 


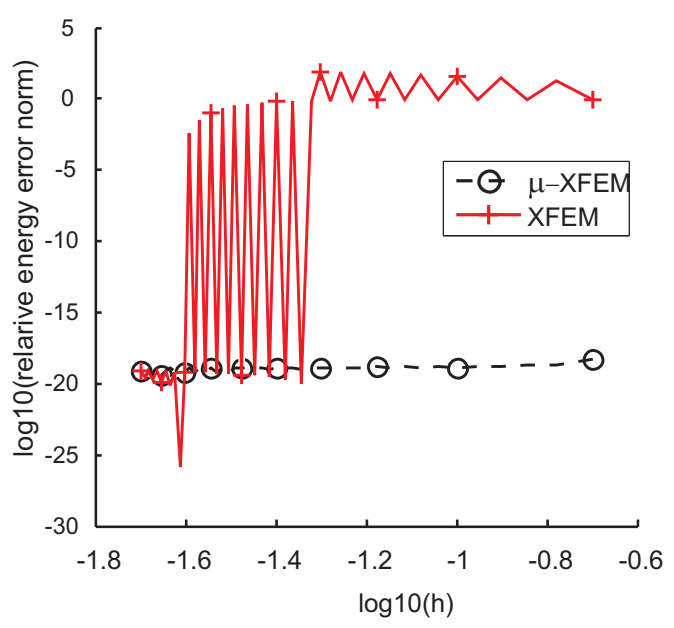

Figure 17. Convergence analysis for the interphase patch test using a regular mesh.

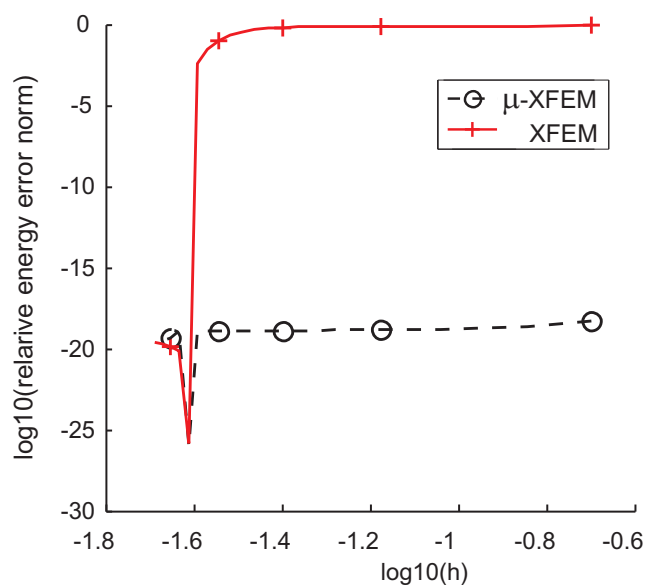

a)

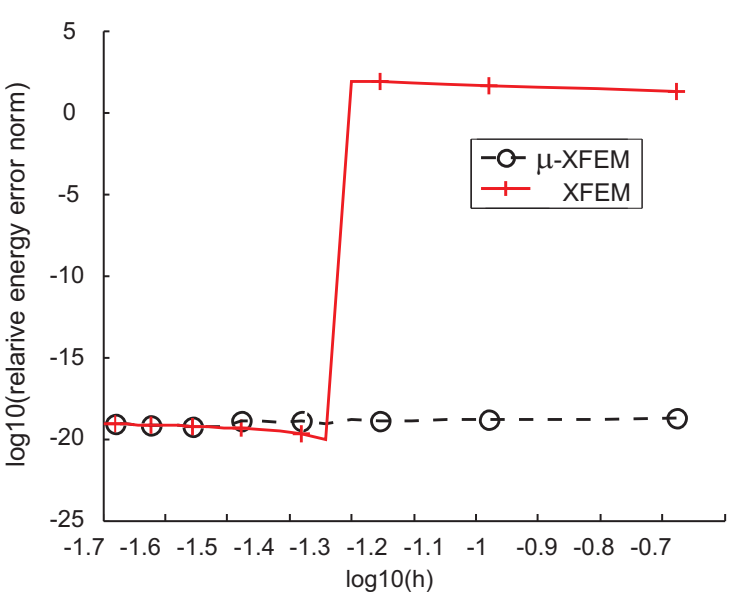

b)

Figure 18. Convergence analysis for the interphase patch test using a regular mesh: a) $n$ odd; b $n$ even.

\subsection{Two dimensional benchmark with nearby inclusions}

We consider a square domain with 4 circular inclusions in a matrix, close to each other. The geometry of the problem is depicted in figure 20. The objective here is to show that in a problem involving nearby inclusions, $\mu$-XFEM convergence is enhanced compared to XFEM. A mesh of linear triangles is used, that does not coincide with the interfaces. With XFEM/level-set, a single level-set function defined by (9) is employed. For $\mu$-XFEM, each inclusion is defined by a level-set function defined by (29). The materials forming the matrix and inclusions are all assumed linear isotropic. The following numerical parameters are chosen: $E_{m}=20 \mathrm{MPa}$, $\nu_{m}=0, E_{i}=2000 \mathrm{MPa}, \nu_{i}=0$, the indices $i$ and $m$ being related to the inclusions and matrix, 


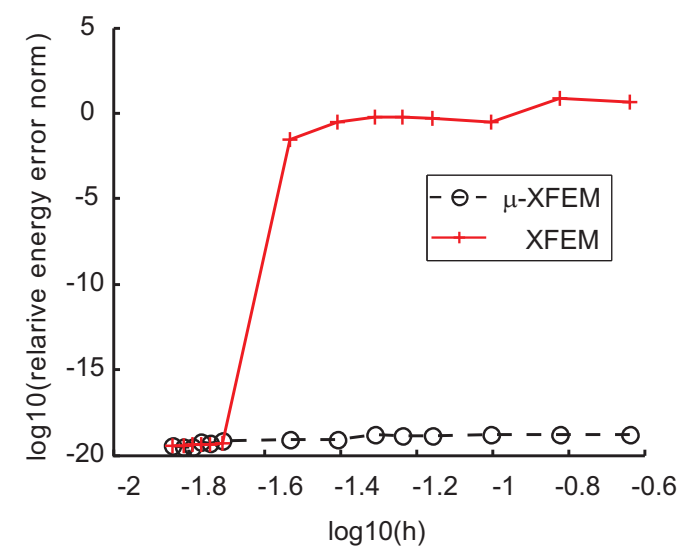

Figure 19. Convergence analysis for the interphase patch test using an unstructured mesh.

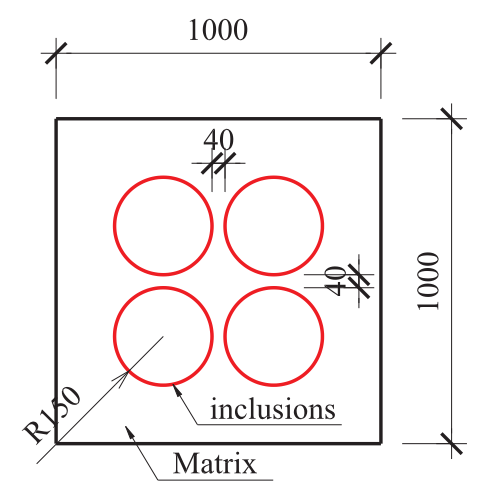

Figure 20. Domain with 4 nearby circular inclusions.

respectively. We compute the homogenized elastic tensor $\overline{\mathbb{C}}$ by prescribing linear displacement conditions and using the classical homogenization procedure [12].

For this problem, an analytical solution is not available. We first study the convergence of the values of the $\bar{C}_{1111}$ component, plotted in figure 21 a) with respect to the mesh size. A reference solution is obtained by using a fine conforming mesh of triangular elements (24898 d.o.f.) which gives $\bar{C}_{1111}^{\text {ref }}=34.7244 \mathrm{MPa}$. The relative error norm of $\bar{C}_{1111}$ defined by:

$$
e=\frac{\left|\bar{C}_{1111}-\bar{C}_{1111}^{r e f}\right|}{\bar{C}_{1111}^{r e f}}
$$

is then analyzed as illustrated in the figure $21 \mathrm{~b}$ ).

We can notice that the $\mu$-XFEM solution converges rather quickly to the reference solution, while the XFEM/level-set method leads to strongly oscillating and slower convergence rate. Both curves coincide when the mesh is fine enough. 


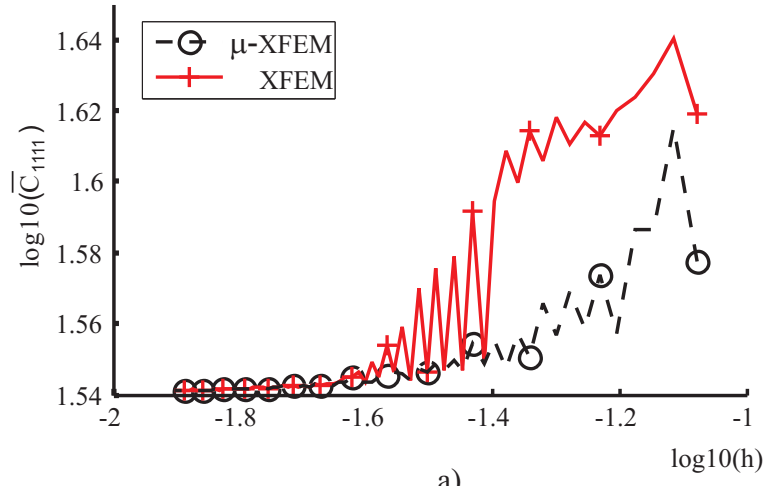

a)

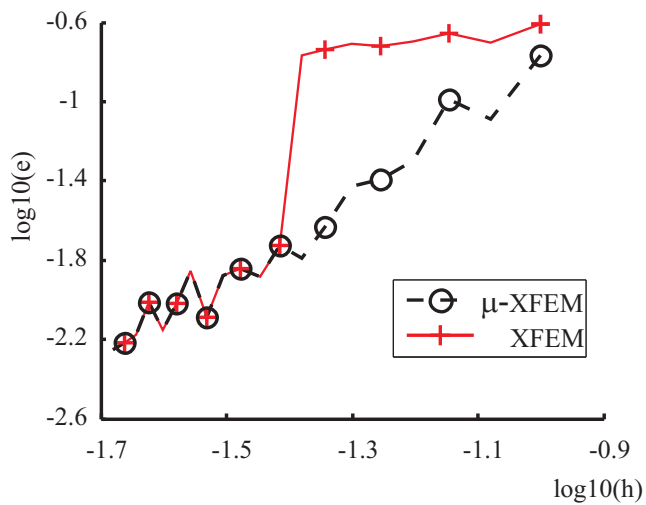

Figure 21. a) Convergence of $\bar{C}_{1111}$ component and b) relative error norm of $\bar{C}_{1111}$ with respect to the mesh size, comparison between XFEM and $\mu$-XFEM.

\section{3. $2 D$ example with 100 inclusions}

In this example, an RVE including 100 circular inclusions is considered and depicted in figure 22 a). The radii are randomly generated, with a uniform probability distribution between $r_{\min }=$ $19.76 \mathrm{~mm}$ and $r_{\max }=59.26 \mathrm{~mm}$. The minimum distance between inclusions is $5.897 \mathrm{~mm}$. The volume fraction of inclusions is $f=0.3703$. Inclusions centers are randomly generated with a non penetration algorithm. A mesh of linear triangles not matching the interfaces is used for both XFEM and $\mu$-XFEM computations.

The inclusion and matrix materials are assumed linearly elastic and isotropic with $E_{m}=1$ $\mathrm{MPa}, \nu_{m}=0.2, E_{i}=100 \mathrm{MPa}, \nu_{i}=0.2$. We compute the effective elasticity tensor $\overline{\mathbb{C}}$ by applying linear boundary conditions related to a constant strain field on the boundary. We study the convergence of $\bar{C}_{1111}$ with respect to the mesh size in figure 23 a) for the both methods. For a fair comparison, the values are plotted with respect to the same number of degrees of freedom. The solution denoted by FEM is obtained via a standard FEM calculation with a mesh conforming to the interfaces and 129540 d.o.f.. The relative error norm defined 


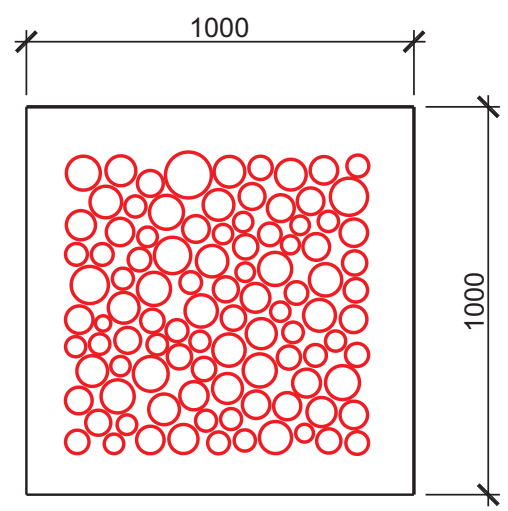

Figure 22. RVE with 100 circular inclusions.

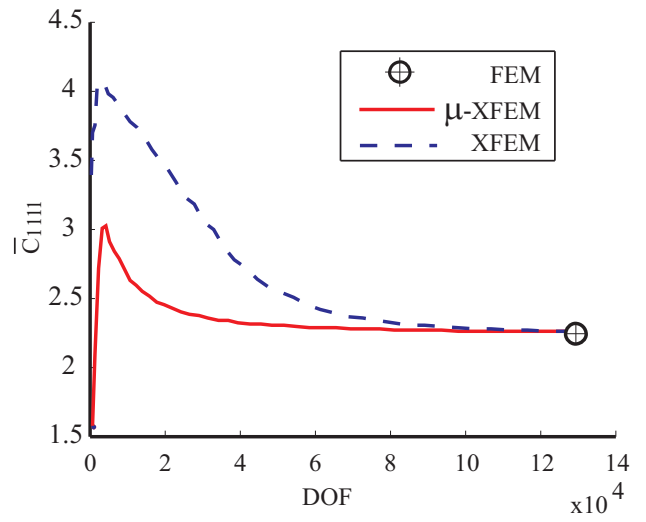

a)

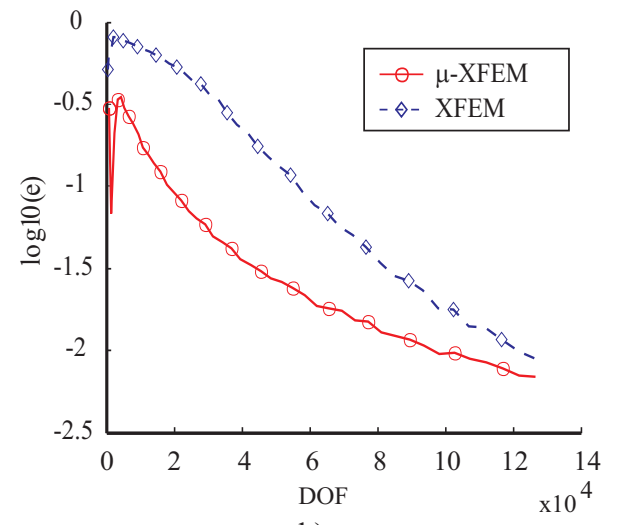

b)

Figure 23. a) Convergence of $\bar{C}_{1111}$, b) the relative error norm of $\bar{C}_{1111}$ versus the total number of d.o.f..

in (58) is also studied and depicted in figure $23 \mathrm{~b}$ ).

We notice in figures $23 \mathrm{a}$ ) and b) that the $\mu$-XFEM solution converges much faster to the reference solution than XFEM. Though this method introduces a higher number of d.o.f. as compared to XFEM, the gain in accuracy is significant for a given number of d.o.f..

\subsection{Three-dimensional RVE}

6.4.1. RVE containing 50 inclusions In this example, a three-dimensional RVE of idealized concrete material is studied. Inclusions are taken to be spherical for the sake of simplicity. A unit cubic domain contains 50 inclusions with random positions, as depicted in figure 24 . The radii of inclusions are randomly generated from a probability function deduced from a practical sieving curve (provided by Electricité De France), with minimal and maximal radii $r_{\min }=0.0215 \mathrm{~m}$ and $r_{\max }=0.0768 \mathrm{~m}$. The total volume fraction of inclusions is $f=0.4194$. Though simplified and idealized in many aspects as compared to the real-life concrete material, 


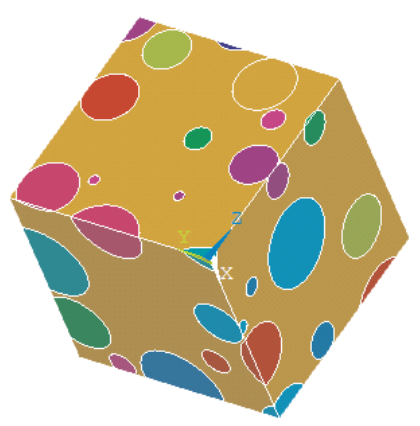

a)

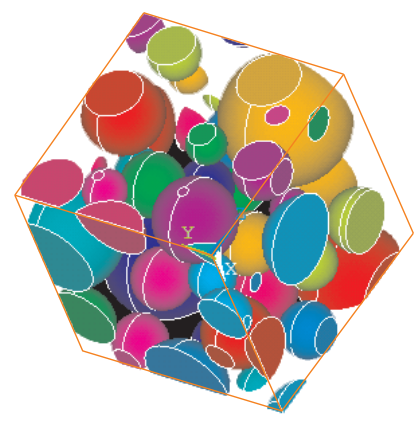

b)

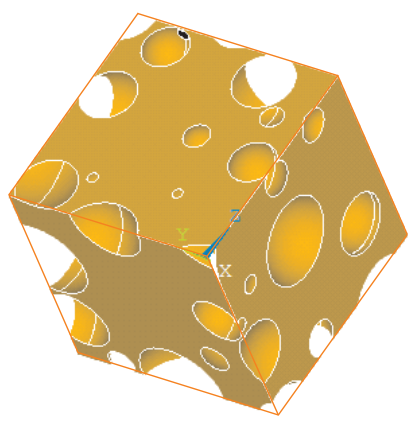

c)

Figure 24. a) RVE containing 50 inclusions; b) the inclusions of RVE; c) the matrix of RVE.

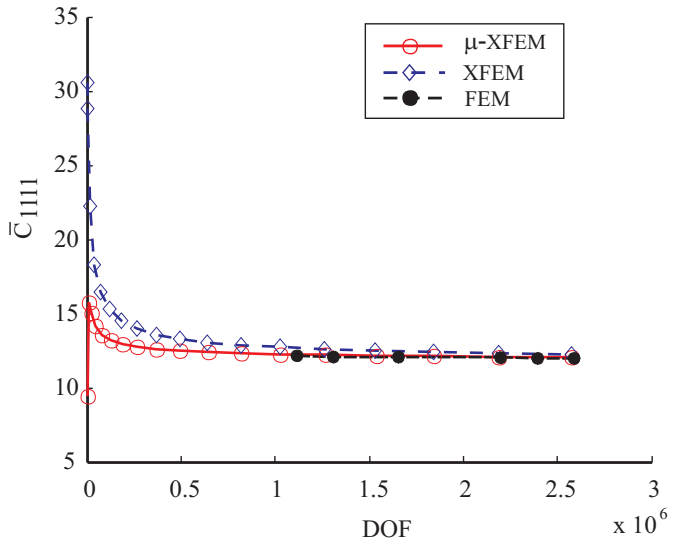

a)

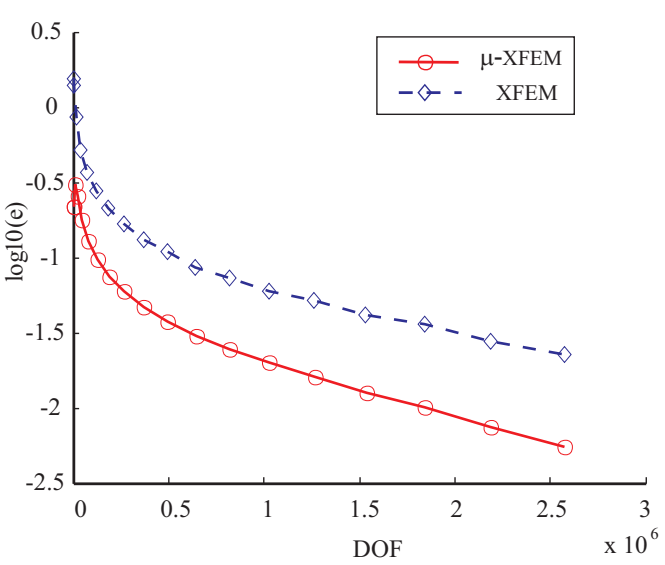

b)

Figure 25. 3D RVE containing 50 inclusions: a) convergence of $\bar{C}_{1111}$; b) the relative error norm of $\bar{C}_{1111}$ with respect to the total number of d.o.f..

this model constitutes a difficult problem, due to the presence of many nearby inclusions. The purpose of this example is to compute the effective elasticity tensor $\overline{\mathbb{C}}$ associated with the homogenized material. We use the classical XFEM/Level-set, $\mu$-XFEM, and a fine 3D FEM model with conforming meshes at the interfaces, for comparison.

Both the matrix and inclusion materials are assumed to be linearly elastic and isotropic with the Young moduli and Poisson ratios $E_{m}=1 \mathrm{MPa}, \nu_{m}=0.2, E_{i}=100 \mathrm{MPa}, \nu_{i}=0.2$.

The cubic domain is meshed with a regular mesh of tetrahedra not conforming with the interfaces. The effective elastic tensor is computed as mentioned in the former example. For this purpose, the additional d.o.f. of nodes which lie on the external boundary of the domain are prescribed to zero for both XFEM and $\mu$-XFEM. A convergence analysis is carried out, using different meshes with increasing elements densities associated with a characteristic size $h$. For each mesh, the effective elastic tensor $\overline{\mathbb{C}}$ is computed.

As no exact solution exists for this problem, we use as a reference solution fine FEM models 
Table I. Number of d.o.f. required to reach a given target error: comparison beteween XFEM and $\mu$-XFEM for the 3D problem with 50 inclusions

\begin{tabular}{lll}
\hline Target error & XFEM (d.o.f.) & $\mu$-XFEM (d.o.f.) \\
\hline $5.0 \%$ & 1294156 & 341289 \\
$2.5 \%$ & 2369454 & 791259 \\
\hline
\end{tabular}

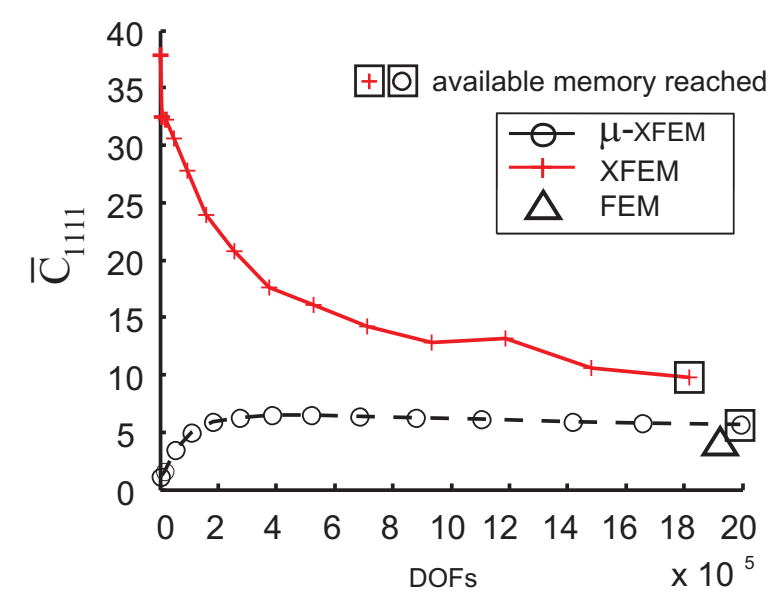

Figure 26. Convergence of effective elastic properties for the 3D RVE containing 2024 inclusions. Both XFEM and $\mu$-XFEM solutions are compared for same number of d.o.f. The FEM solution has been obtained by using a conforming tetrahedral mesh to the interfaces and 9 million d.o.f.

with meshes of tetrahedra conforming to the interfaces. The reference solution corresponds to a converged value with respect to several FEM mesh densities, as shown in figure 25 a). The convergence analysis of component $\bar{C}_{1111}$ is shown in figure $25 \mathrm{~b}$ ). We can notice that while both XFEM and $\mu$-XFEM converge to the reference solutions, the $\mu$-XFEM converges faster. In table $\mathrm{I}$, we compare the number of degrees of freedom required to reach a given relative error on $\bar{C}_{1111}$. We can note that for an error of $5 \%$ the gain using $\mu$-XFEM over X-FEM is 3.7 and for $2.5 \%$ error 2.99 .

6.4.2. RVE containing 2024 inclusions In this second test, the unit cube defining the RVE contains 2024 inclusions. As previously, the radii of inclusions are randomly generated with minimal and maximal radii $r_{\min }=0.0214 \mathrm{~m}$ and $r_{\max }=0.0833 \mathrm{~m}$. The total volume fraction of inclusions is $f=0.4$. The mechanical model for the different phases is the same as in the previous test. For that case, constructing a conforming mesh is a very difficult task. For this reason, only one FEM solution was computed. It contains $9.10^{6}$ d.o.f and was provided y B. Bary, CEA, France [2]. As we do not have other solution for finer meshes, we can not conclude that this FEM solution is a reference solution, it is in this example only indicative. In figure 26 , we show the convergence analysis of the component $\bar{C}_{1111}$. We note that the $\mu$-XFEM converges faster than XFEM, as a function of the total number of d.o.f.

In figure 27 we show the interfaces approximated by the single level-set and multiple level-set methods, for a mesh corresponding to 511104 elements. Qualitatively, it can be observed that $\mu$-XFEM avoids the artificial percolation. As it also removes the other artefacts, the faster 


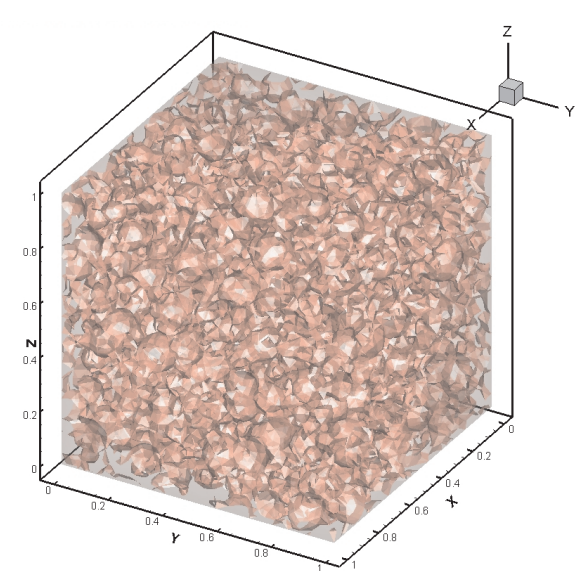

a)

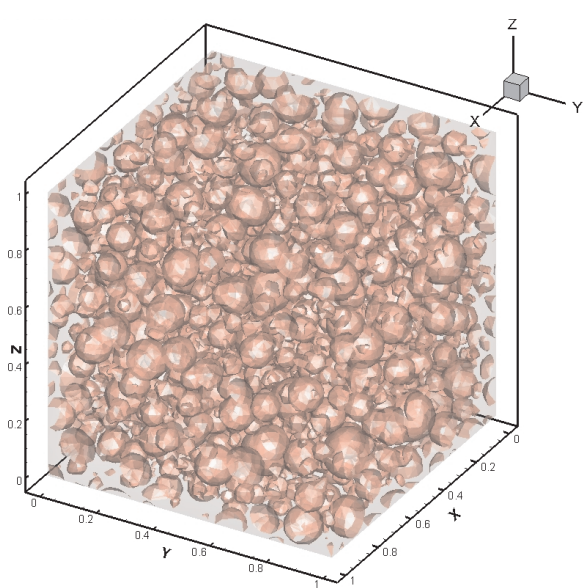

b)

Figure 27. Approximated interfaces for the 3D model of concrete RVE: a) level-set; b) multiple levelset; the same mesh with 511104 elements were used in both cases.

convergence of this method as compared to XFEM/level-set can be explained.

\section{CONCLUSIONS}

We have shown in this paper that when microstructures contain high volume fractions of inclusions or very nearby inclusions, the use of XFEM/level-set method for the numerical analysis induces numerical artefacts which affect significantly accuracy and convergence of the solution. The first two artefacts are related to the level-set approximation: they induce artificial percolation between inclusions and additional distortion of interfaces. The third one is related to the enriched approximation scheme, which fails to reproduce a piece-wise linear field within connected elements, if each of these elements contains at least one interface. We have proposed a method to avoid these issues: each inclusion is associated to its own level-set and additional d.o.f. are introduced. We have shown that the proposed approach avoids the aforementioned issues and compared the gains with XFEM/level-set through different benchmark problems. The results indicate that in applications aiming at determining the effective properties of complex heterogeneous materials with high volume fractions of inclusions, the convergence with respect to the mesh size is significantly enhanced, allowing the use of much coarser meshes to predict the effective properties. Another advantage of this approach is the capacity of treating the case where each phase is endowed with its own properties.

\section{ACKNOWLEDGEMENTS}

The support this work enjoys from EDF is gratefully acknowledged. We thank B. Bary, Commissariat à l'Energie Atomique (CEA), France, for providing us the large 3D FEM solution. 


\section{REFERENCES}

1. F. Barbea, R. Queyc, A. Musienkod, G. Cailletaud (2009). Three-dimensional characterization of strain localization bands in high-resolution elastoplastic polycrystals. Mech. Res. Commun., 36(7):762-768.

2. B. Bary, M. Ben Haha, E. Adam, P. Montarnal (2009). Numerical and analytical effective elastic properties of degraded cement pastes. Cem. Concr. Res., 39(10):902-912.

3. E. Béchet, N. Moës, B. Wohlmuth (2009). A stable Lagrange multiplier space for stiff interface conditions within the extended finite element method. Int. J. Numer. Methods Eng., 78:931-954.

4. T. Belytschko, T. Black (1999). Elastic crack growth in finite elements with minimal remeshing. Int. J. Numer. Methods Eng., 45(5):601-620.

5. T.Belytschko, C. Parimi, N. Moës, N. Sukumar, S. Usui (2003). Structured extended Finite element methods for solids defined by implicit surfaces. Int. J. Numer. Methods Eng., 56:609-635.

6. C. Daux, N. Moës, J. Dolbow, N. Sukumar, T. Belytschko (2000). Arbitrary branched and intersecting cracks with the extended finite element method. Int. J. Numer. Methods Eng., 48(12):1741-1760.

7. M.P. Do Carmo (1976). Differential Geometry of Curves and Surfaces. Prentice-Hall, New-Jersey.

8. C. Duarte, L.G. Reno, A. Simone. (2007). A high-order generalized FEM for through-the-thickness branched cracks. Int. J. Numer. Methods Eng., 72:325-351.

9. T.P. Fries (2008). A corrected XFEM approximation without problems in blending elements. Int. J. Numer. Methods Eng., 75(5):503-32.

10. R. Gracie, H. Wang, T. Belytschko (2008). Blending in the extended finite element method by discontinuous Galerkin and assumed strain methods. Int. J. Numer. Methods Eng., 74(11):1645-669.

11. H. Ji, J.E. Dolbow (2004). On strategies for enforcing interfacial constraints and evaluating jump conditions with the extended finite element method. Int. J. Numer. Methods Eng., 61:2508-2535.

12. T. Kanit, S. Forest, I. Galliet, V. Mounoury, D. Jeulin (2003). Determination of the size of the representative volume element for random composites: statistical and numerical approach. Int. J. Solids Struct., 40:3647-3679.

13. B.L. Karihaloo, Q.Z. Xiao (2003). Modelling of stationary and growing cracks in FE framework without remeshing: a state-of-the-art review. Comput. and Struct., 81:119-129.

14. J.M. Melenk, I. Babuška (1996). The partition of unity Finite element method: basic theory and applications. Comput. Meth. Appl. Mech. Eng., 39:289-14.

15. R. Merle, J. Dolbow (2002). Solving thermal and phase change problems with the eXtended finite element method. Comput. Mech., 28(5):339-350.

16. N. Moës, J. Dolbow, T. Belytschko (1999). A finite element method for crack growth without remeshing. Int. J. Numer. Methods Eng. 46(1):131-156.

17. N. Moës, M. Cloirec, P. Cartraud, J.-F. Remacle (2003). A computational approach to handle complex microstructure geometries. Comput. Meth. Appl. Mech. Eng., 192:3163-3177.

18. N. Moës, E. Bechet, M. Tourbier (2006). Imposing Dirichlet boundary conditions in the extended finite element method. Int. J. Numer. Methods Eng., 67:1641-1669.

19. H.M. Mourad, J. Dolbow, I. Harari (2007). A bubble-stabilized finite element method for Dirichlet constraints on embedded interfaces. Int. J. Numer. Methods Eng., 69:772-793.

20. S. Osher, J.A. Sethian (1998). Fronts propagating with curvature-dependent speed: Algorithms based on Hamilton-Jacobi formulations. J. Comput. Phys. 79(1):12-49.

21. N. Sukumar, D.L. Chopp, N. Moës, T. Belytschko (2001). Modeling holes and inclusions by level-sets in the extended finite-element method. Int. J. Solids Struct., 190:6183-6200.

22. N. Sukumar, N. Moës, B. Moran, T. Belytschko (2000). Extended finite element method for threedimensional crack modelling. Int. J. Numer. Methods Eng., 48(11):1549-1570.

23. J.A. Thorpe (1979). Elementary Topics in Differential Geometry. Springer-Verlag, Berlin.

24. C. Toulemonde, R. Masson, J. El Gharib (2008). Modeling the effective elastic behavior of composites: a mixed finite element and homogenisation approach. C. R. Mec., 336(3):275-282.

25. J. Yvonnet, H. Le Quang, Q.-C. He (2008). An XFEM/level-set approach to Modeling surface/interface effects and to computing the size-dependent effective properties of nanocomposites. Comput. Mech., 42:119131.

26. J. Yvonnet, Q.-C. He, C. Toulemonde (2008). Numerical modelling of the effective conductivities of composites with arbitrarily shaped inclusions and highly conducting interface. Compos. Sci. Technol., 68:2828-2825.

27. J. Yvonnet, Q.-C. He, Q. Zhu, J.-F. Shao, (2010). A general and efficient computational procedure for modelling the Kapitza thermal resistance based on XFEM. Comput. Mat. Sci., in press, doi:10.1016/j.commatsci.2010.02.040.

28. T.I. Zohdi, P. Wriggers (2001). Aspects of the computational testing of the mechanical properties of microheterogeneous material samples. Int. J. Numer. Methods Eng., 50:2573-2599. 